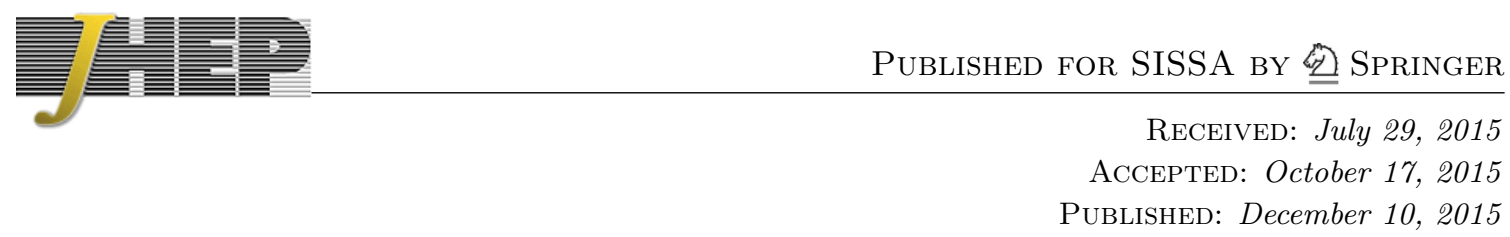

\title{
Superconformal indices and M2-branes
}

\author{
Richard Eager ${ }^{a}$ and Johannes Schmude ${ }^{b}$ \\ ${ }^{a}$ Kavli Institute for the Physics and Mathematics of the Universe (WPI), \\ University of Tokyo, Kashiwa, Chiba 277-8583, Japan \\ ${ }^{b}$ RIKEN Nishina Center, Saitama 351-0198, Japan \\ E-mail: richard.eager@ipmu.jp, johannes.schmude@riken.jp
}

ABSTRACT: We derive the superconformal index of the world-volume theory on M2-branes probing the cone over an arbitrary Sasaki-Einstein seven-manifold. The index is expressed in terms of the cohomology groups of the cone. We match our supergravity results with known results from gauge theory. Along the way we derive the spectrum of short KaluzaKlein multiplets on generic Sasaki-Einstein seven-manifolds.

KEYwords: AdS-CFT Correspondence, Supersymmetric gauge theory, M-Theory

ARXIV EPRINT: 1305.3547 


\section{Contents}

1 Introduction $\quad 2$

2 Calculation of the index 3

2.1 Unitary representations of the $\mathcal{N}=2$ superconformal algebra 5

2.2 The short graviton multiplet 5

$\begin{array}{lll}2.3 & \text { The short gravitino multiplet } & 6\end{array}$

$\begin{array}{ll}2.4 & \text { The short vector and Betti multiplets }\end{array}$

$\begin{array}{lll}2.5 & \text { Hypermultiplets } & 7\end{array}$

$\begin{array}{lll}3 & \text { Cohomology calculations } & 7\end{array}$

$\begin{array}{lll}3.1 \text { Toric Calabi-Yau varieties } & 7\end{array}$

3.2 Toric examples 8

$\begin{array}{ll}3.3 \text { Cones over Fano varieties } & 10\end{array}$

3.4 Twisted cohomology of Fano varities 11

$\begin{array}{ll}3.5 & \text { The seven-sphere } S^{7} \\ 3.6 & 12\end{array}$

$\begin{array}{ll}3.6 & \text { The Stiefel manifold } V_{5,2} \\ \end{array}$

4 The supergravity analysis $\quad 14$

$\begin{array}{lll}4.1 & \text { The Kaluza-Klein spectrum } & 14\end{array}$

$\begin{array}{lll}4.2 & \text { The multiplets } & 17\end{array}$

4.3 Previous work on Kaluza-Klein compactification 22

5 Conclusions 23

$\begin{array}{ll}\text { A Conventions and useful expressions } & 24\end{array}$

$\begin{array}{ll}\text { B Details of the supergravity analysis } & \mathbf{2 7}\end{array}$

$\begin{array}{ll}\text { B.1 Wave functions constructed from scalars } & 27\end{array}$

$\begin{array}{lll}\text { B.2 Wave functions derived from one-forms } & 29\end{array}$

$\begin{array}{ll}\text { B.3 Wave functions derived from two-forms } & 31\end{array}$

B.4 Additional three-form modes 32

C Comments on Lefschetz decomposition and Kohn-Rossi cohomology 33

D Cohomology using Borel-Weil-Bott 34 


\section{Introduction}

The superconformal index of a three-dimensional superconformal field theory is the partition function of the theory on $S^{1} \times S^{2}$ with supersymmetric boundary conditions. Equivalently, the index can be expressed as the trace over all operators in the theory weighted by their fermion number

$$
\mathcal{I}\left(t, z_{i}\right)=\operatorname{Tr}\left[(-1)^{F} t^{\epsilon+j_{3}} z_{i}^{h_{i}}\right] .
$$

Here $\epsilon$ is the operator dimension, $j_{3}$ is the spin of the operator, $F$ is its fermion number, and $h_{i}$ label the charges of the operator under global symmetries. The superconformal index is invariant under exactly marginal deformations and can be computed from an ultraviolet Lagrangian description, provided that the infrared R-symmetry can be identified in the ultraviolet [1]. The superconformal index was originally defined for four dimensional superconformal field theories [2,3] and was generalized to three dimensional theories in [4].

A large class of three dimensional superconformal field theories is realized as the low energy effective theory of multiple M2-branes probing a Calabi-Yau fourfold singularity. These theories have $\mathcal{N}=2$ supersymmetry and have a holographic dual description as M-theory on the product of four-dimensional anti-de Sitter space $A d S_{4}$ and a sevendimensional Sasaki-Einstein manifold. The simplest Calabi-Yau fourfold singularity is $\mathbb{C}^{4} / \mathbb{Z}_{k}$. The theory of M2-branes at this singularity is realized as the low energy limit of a quiver Chern-Simons theory with $\mathcal{N}=8$ supersymmetry when $k=1,2$ [5]. The holographic dual theory is M-theory on $A d S_{4} \times S^{7} / \mathbb{Z}_{k}$ and the supergravity index was computed by summing all contributions from short multiplets [4]. The field theory and supergravity superconformal indices were shown to match in the large $k$ limit [6]. At finite $k$, monopole operators contribute to the index and their contribution can be computed using localization $[7,8]$.

In this paper we will derive the gravity superconformal index for any theory of the form $A d S_{4} \times S E_{7}$. Previously the supergravity index was computed for the homogenous Sasaki-Einstein seven-manifolds using known Kaluza-Klein spectra [9]. However, to match the field theory index and the supergravity index, several of the Kaluza-Klein modes in [10] had to be dropped. Since the spectrum has not been well tested, the authors suggested that the Kaluza-Klein spectrum should be revisited. We find that a careful analysis of the Kaluza-Klein modes agrees with the field theory index $[9,11,12]$. Our general form of the supergravity index succinctly reproduces previous computations of the gravity index [9]. We find complete agreement with previous large- $N$ computations of the index $[9,11,12]$.

We construct the Kaluza-Klein multiplets on $A d S_{4}$ from various tensors defined on the Sasaki-Einstein manifold following the methodology of [13]. Our analysis focuses on generic Sasaki-Einstein manifolds. Much of our analysis builds upon previous work on Kaluza-Klein spectroscopy for coset manifolds [10, 14-16]. The structure of the KaluzaKlein multiplets is intimately related to the spectrum of various differential operators on the internal manifold. For manifolds with coset structure the spectral problems were solved using harmonic analysis, which is not applicable to generic Sasaki-Einstein manifolds. However, supersymmetry implies relations between the masses of Kaluza-Klein modes and thus also between the eigenmodes of the differential operators on the internal manifold [15]. The 
approach of [13] exploits these relations to reconstruct the general form of the Kaluza-Klein multiplets in terms of the Sasakian structure. ${ }^{1}$ Recall that any Sasaki-Einstein manifold admits a conformal Killing spinor $\Theta$ satisfying $\nabla_{V} \Theta=\frac{1}{2} V \cdot \Theta$ for any vector field $V$. Given an eigenfunction of the Laplace, Dirac or Lichnerowicz operator, one can obtain further eigenmodes by repeatedly tensoring with $\Theta$ and acting with $\nabla$. If one wants to consider only bosonic modes, one considers $\nabla$ in conjunction with the tensors that arise as bilinears of the spinor instead of $\Theta$ itself. This is the approach that we will follow in this paper. It allows us to obtain the complete structure of the Kaluza-Klein multiplets, identify the short multiplets, and calculate the index.

Multiplet shortening and the short multiplets contributing to the index can be described using the tangential Cauchy-Riemann operator $\bar{\partial}_{B}$ on the Sasaki-Einstein manifold $Y$ and the associated Kohn-Rossi cohomology groups $H_{\bar{\partial}_{B}}^{p, q}$. In general, the cotangent bundle over $Y$ can be decomposed as

$$
\Omega_{Y}=\mathbb{C} \eta \oplus \Omega_{Y}^{1,0} \oplus \Omega_{Y}^{0,1}
$$

The operator $\bar{\partial}_{B}$ is the projection of the exterior derivative on $\Omega_{Y}^{0,1}$, the cohomology of this complex is $H_{\bar{\partial}_{B}}^{p, q}[18,19]$. The Kohn-Rossi cohomology groups are isomorphic to $H^{q}\left(X, \wedge^{p} \Omega_{X}^{\prime}\right)$ defined on the cone $X$ over $Y$, where $\Omega_{X}^{\prime}$ is the part of the holomorphic cotangent bundle $\Omega_{X}$ perpendicular to the dilatation vector field. Our main result is a formula for the gravity superconformal index as a trace over linear combinations of the groups $H^{q}\left(X, \wedge^{p} \Omega_{X}^{\prime}\right)$.

Organization. The organization of this paper is as follows: in section 2 we calculate the single trace index drawing on the results from the remaining sections of this paper. Section 3 shows how the trace over cohomology groups can be evaluated for toric and Fano manifolds. The Kaluza-Klein analysis of Sasaki-Einstein seven-manifolds used in section 2 is performed in section 4, with technical details refered to the appendices A and B. Section 4.3 describes relations between this paper and previous work on Kaluza-Klein spectroscopy.

\section{Calculation of the index}

In this section we list the multiplicity of each short multiplet appearing in supergravity solutions of the form $A d S_{4} \times S E_{7}$ and their contribution to the superconformal index. The single trace superconformal index is defined by the following modification of (1.1)

$$
\mathcal{I}_{\text {s.t. }}\left(t, z_{i}\right)=\operatorname{Tr}_{\text {s.t. }}\left[(-1)^{F} t^{\epsilon+j_{3}} z_{i}^{h_{i}}\right] \text {. }
$$

Only states with

$$
\{Q, S\}=\epsilon-j_{3}-y=0
$$

contribute, where $y$ is the R-charge.

The general method to calculate the index from holography is quickly summarized. Using the AdS/CFT dictionary one has to identify all states contributing to (2.1). Single

\footnotetext{
${ }^{1}$ For a similar analysis in the context of Kähler geometry see [17].
} 
trace operators in field theory are dual to supergravity modes in $A d S_{4}$. These modes on the other hand arise from linearized fluctuations around the full eleven-dimensional $A d S_{4} \times S E_{7}$ solution. The quantum numbers of the fluctuations are determined by the eigenvalues of various differential operators on the internal Sasaki-Einstein manifold. Consider for example an operator $\mathcal{O}$ with conformal dimension $\Delta$. The AdS/CFT dictionary relates the conformal dimension $\Delta$ to the mass of a fluctuation in $A d S_{4}$; see table 20. For $k$-form fields with mass $m$, the relation is $m^{2}=\Delta(\Delta-3+k)$. Standard Kaluza-Klein analysis determines these masses in terms of the eigenvalues of the Laplace, Dirac, or Lichnerowicz operators on the internal $S E_{7}$ manifold [20]. The R-charge and spin, which also enter in (2.1), follow from the charge of the mode under translation along the Reeb vector or directly from the AdS/CFT dictionary respectively. Since the Kaluza-Klein analysis for space-times of the form $A d S_{4} \times Y$ with $Y$ Sasaki-Einstein has been previously performed in a general setting [20,21], the problem of evaluating (2.1) from supergravity reduces to that of obtaining the full spectrum of the Laplace (Dirac, Lichnerowicz) operators on the $S E_{7}$ manifold. We do so in section 4 and appendix B.

The results of this analysis can be found in tables 8 to 11 that list the eigenmodes of the Laplace operator on $p$-forms on $S E_{7}$. Using tables 20 and 21 one then obtains the general form of the Kaluza-Klein spectrum of the linearized fluctuations as well as the scaling dimension of the dual operators. This determines the multiplets, which are listed in the tables tables 12 to 19. The explicit calculations done in this paper deal only with bosonic modes, yet as the structure of the multiplets is fully determined by the superconformal algebra, fermionic modes are fully determined by this. In order to keep the tables short, we have only included those relevant for the calculation of the index. The names appearing in these tables denote the origin of a mode in regard to the Kaluza-Klein analysis. We use the same notation as in [16]. It can happen that various wavefunctions in the tables 8 to 11 vanish. This leads to a vanishing of the corresponding modes in 12 to 19 - i.e. to short multiplets.

Short multiplets contributing to the index and their multiplicities are listed in table 1. An element $f$ of cohomology has R-charge $\mathcal{L}_{D} f=2 i D f$. Here $\mathcal{L}_{D}$ denotes the Lie derivative along the dilation vector field and $2 D$ is its corresponding eigenvalue. We normalize each multiplet so that its primary has R-charge $y$. The R-charge $y$ differs from the R-charge $2 D$ of the corresponding cohomology element by a constant shift.

A short multiplet whose primary has quantum numbers $\left(y+j_{3}+1, j_{3}, y\right)$ contributes $(-1)^{2 j_{3}+1} t^{y+2 j_{3}+2}$ to the index. The hypermultiplets with quantum numbers $(y, 0, y)$ contribute $t^{y}$ to the index. Summing the contributions of the short multiplets, we find that the single particle supergravity index is

$$
\begin{aligned}
1+\mathcal{I}_{\text {s.t. }}(t)=\sum \operatorname{Tr} t^{2 D} \mid H^{0}\left(X, \mathcal{O}_{X}\right) \ominus H^{0}\left(X, \wedge^{2} \Omega_{X}^{\prime}\right) \oplus H^{1}\left(X, \wedge^{2} \Omega_{X}^{\prime}\right) \\
\\
\quad \oplus t^{2} H^{0}\left(X, \Omega_{X}^{\prime}\right) \ominus t^{2} H^{1}\left(X, \Omega_{X}^{\prime}\right) \oplus t^{2} H^{2}\left(X, \Omega_{X}^{\prime}\right) \ominus t^{2} H^{0}\left(X, \wedge^{3} \Omega_{X}^{\prime}\right) .
\end{aligned}
$$

Here, $\operatorname{Tr} A \mid V$ denotes the trace of an operator $A$ acting on a vector space $V$. The single particle index is similar to the single-trace index, but it also includes the derivatives of the 


\begin{tabular}{|l|c|l|c|c|}
\hline Multiplet & Primary $\left(\epsilon, j_{3}, y\right)$ & Multiplicity & Index & Index \\
\hline short graviton & $(y+2,1, y)$ & $H^{0}\left(X, \wedge^{3} \Omega_{X}^{\prime}\right)$ & $-t^{y+4}$ & $-t^{2 D+2}$ \\
short gravitino & $\left(y+\frac{3}{2}, \frac{1}{2}, y\right)$ & $H^{0}\left(X, \Omega_{X}^{\prime}\right)$ & $t^{y+3}$ & $t^{2 D+2}$ \\
short vector $Z /$ betti & $(y+1,0, y)$ & $H^{1}\left(X, \Omega_{X}^{\prime}\right)$ & $-t^{y+2}$ & $-t^{2 D+2}$ \\
short vector $A$ & $(y+1,0, y)$ & $H^{0}\left(X, \wedge^{2} \Omega_{X}^{\prime}\right)$ & $-t^{y+2}$ & $-t^{2 D}$ \\
hyper & $(y, 0, y)$ & $H^{1}\left(X, \wedge^{2} \Omega_{X}^{\prime}\right)$ & $t^{y}$ & $t^{2 D}$ \\
hyper & $(y, 0, y)$ & $H^{2}\left(X, \Omega_{X}^{\prime}\right)$ & $t^{y}$ & $t^{2 D+2}$ \\
hyper & $(y, 0, y)$ & $H^{0}\left(X, \mathcal{O}_{X}\right)$ & $t^{y}$ & $t^{2 D}$ \\
\hline
\end{tabular}

Table 1. Short multiplets and their contribution to the index.

single-trace operators. These two indices are related by ${ }^{2}$

$$
\mathcal{I}_{\text {s.t. }}(t)=\left(1-t^{2}\right) \mathcal{I}_{\text {s.p. }}(t) .
$$

We consider only Sasaki-Einstein manifolds. For Sasaki-Einstein spaces with singularities, there can be additional contributions [22,23]. We next explain how the multiplet shortening conditions arise from the supergravity spectrum after reviewing the structure of short and long multiplets.

\subsection{Unitary representations of the $\mathcal{N}=2$ superconformal algebra}

Supergravity on $A d S_{4} \times S E_{7}$ has $\mathcal{N}=2$ superconformal symmetry. We begin by recalling the properties of the three dimensional $\mathcal{N}=2$ superconformal group $O s p(2 \mid 4)$. Its bosonic subgroup is $\operatorname{Sp}(4, \mathbb{R}) \oplus \mathrm{SO}(2)_{R}$. The first factor $\operatorname{Sp}(4, \mathbb{R}) \cong \mathrm{SO}(2,3)$ is the isometry group of $A d S_{4}$ and the second factor, $\mathrm{SO}(2)_{R}$, is the R-symmetry group.

Unitary representations are labeled by their eigenvalues $\epsilon, j_{3}, y$ under the dilation, angular momentum, and R-symmetry operators. Unitary representations with spin $j_{3}>0$ satisfy $\epsilon-j_{3}-y \geq 0$. Representations saturating this bound have null states and the representation shortens. Hence such representations are called short. Representations not saturating this bound are called long. When $j_{3}=0$ representations satisfying $\epsilon=y$ are called isolated. All other representations with $j_{3}=0$ satisfy $\epsilon \geq y+1$; those saturating this bound are called short.

\subsection{The short graviton multiplet}

The graviton multiplet, shown in table 12, can be constructed starting from a scalar eigenfunction of the Laplacian on the Sasaki-Einstein manifold. The scalar eigenfunction is the wave function of the spin 2 graviton in the multiplet. An eigenvalue $\Delta_{0}$ of the scalar Laplacian is bounded by its charge ${ }^{3} q$ along the Reeb vector $\xi$ via $\Delta_{0} \geq q(q+6)$, with

\footnotetext{
${ }^{2}$ Here we omit the contribution from the identity operator.

${ }^{3} q$ is the eigenvalue of the Lie derivative along $\xi, £_{\xi}$. It is related to the R-charge $y$ via $q=2 y+c$ for some constant $c$ that is different for each multiplet. See the various tables in section 4 .
} 
equality if and only if the eigenfunction lifts to a holomorphic function on the CalabiYau cone. Equivalently, the eigenfunction is holomorphic with respect to the tangential Cauchy-Riemann operator $\bar{\partial}_{B}$. The Lichnerowicz obstruction imposes $q \geq 1$, with equality if and only if the manifold is isometric to $S^{7}[24]$.

If the scalar eigenfunction is holomorphic, a number of wave-functions vanish and the graviton multiplet shortens. Thus, each element $f \in H^{0}\left(X, \mathcal{O}_{X}\right)$ defines a short graviton multiplet. In each multiplet, the scalar eigenfunction $f$ is the wave-function of the graviton with energy $y+3$, spin 2 , and R-charge $y$. However, the primary has energy $y+2$, spin 1 , and R-charge $y$. Within the superconformal multiplet only the mode $\chi^{+}$contributes to the index. It has energy $y+5 / 2$ and spin $3 / 2$, so the net contribution to the index is $(-1) t^{y+4}$. Since $H^{0}\left(X, \mathcal{O}_{X}\right) \cong H^{0}\left(X, \wedge^{3} \Omega_{X}^{\prime}\right)$, we can express the contribution of the short graviton multiplets in terms of either cohomology group. Note however that the map between the two cohomology groups involves the holomorphic volume form, which carries R-charge 2 .

\subsection{The short gravitino multiplet}

The two gravitino multiplets $\chi^{+}$and $\chi^{-}$can be constructed from one-form eigenmodes on the Sasaki-Einstein manifold. These one-forms are the wave-functions of the vector fields $A$ and $W$ respectively. The multiplets are listed in tables 16 and 17 . By comparing the action of $\partial_{B} \bar{\partial}_{B}$ and $J \wedge$ on one-forms, we conjecture that there is a holomorphy bound $\Delta_{1} \geq q(q+4)$, where $y+1=2 q$. This is equivalent to the standard unitarity condition $E_{0} \geq y+\frac{3}{2}$. Its saturation implies that $\chi^{+}$shortens. Thus every element of $H^{0}\left(X, \Omega_{X}^{\prime}\right)$ defines a short gravitino multiplet. The contribution to the index, $t^{y+3}$, comes from the mode $A$ with R-charge $y+1$, spin 1 , and energy $y+2$.

\subsection{The short vector and Betti multiplets}

The short vector multiplet $A$ arises from holomorphic $(2,0)$ forms. Since the wave-function of the primary mode is a scalar, it is convenient to construct all wave-functions in terms of a scalar, as we did in the case of the graviton. The primary has energy and R-charge $E_{0}$ and $y$. The holomorphic volume form $\Omega$ maps scalars $f$ to $(2,0)$ forms by $\left.\bar{\partial}_{B} f\right\lrcorner \Omega$. Since $\Omega$ carries R-charge 2 , the R-charge of the two-form is $y+2$. The holomorphy bound on two-forms can then be expressed in terms of the $\Delta_{0}$ eigenvalue of $f$. Accommodating for the shifted charge, one finds $\Delta_{0}=4 E_{0}\left(E_{0}+3\right) \geq 4(y+1)(y+4)$. The inequality is saturated if the two-form is holomorphic. Then $E_{0}=y+1$ and the multiplet shortens since a number of wave-functions disappear. Hence, the elements of $H^{0}\left(X, \wedge^{2} \Omega_{X}^{\prime}\right)$ correspond to short vector multiplets $A$. For further details see table 13 and the discussion in section 4.2.

Finally we turn to the vector multiplet $Z$, shown in table 15 . It is constructed from primitive $^{4}(1,1)$ forms and shortens when these forms are holomorphic and the bound $\Delta_{1} \geq q(q+2)$ is saturated. The primary is a scalar with energy $E_{0}$, R-charge $y$, and a three-form wave-function. As argued in appendix $\mathrm{C}$, the primitive forms fill out the cohomology group $H_{\bar{\partial}_{B}}^{1,1}$. Therefore the elements of $H^{1}\left(X, \Omega_{X}^{\prime}\right)$ correspond to the short

\footnotetext{
${ }^{4}$ Recall that a form $\omega$ is primitive if it is annihilated by the adjoint of the Lefschetz operator $\left.-J\right\lrcorner \omega=0$.
} 
vector multiplets $Z$. In section 4.2 we will show that $H_{d R}^{2}(Y) \subseteq H^{1}\left(X, \Omega_{X}^{\prime}\right)$. Therefore we find $b_{2}(Y)$ Betti multiplets $[10,15,25-27]$ and possibly additional charged modes. ${ }^{5}$

\subsection{Hypermultiplets}

There are two different sources for hypermultiplets coming from Kaluza-Klein reduction. The first arises from holomorphic scalar eigenfunctions. When the scalar eigenfunctions are holomorphic, the long vector multiplets $A$ shorten into hypermultiplets. Thus, each element of $H^{0}\left(X, \mathcal{O}_{X}\right)$ defines a hypermultiplet. The scalar eigenfunction is the wave-function of the scalar primary, which has energy and charge $y$. The hypermultiplets contribute $t^{y}$ to the index.

A second source for hypermultiplets are primitive, holomorphic $(2,1)$ and $(1,2)$ forms on the Sasaki-Einstein manifold (table 19). Here, the holomorphy bound is $\Delta \geq q^{2}$. Their contribution to the index is also $t^{y}$. It follows from our arguments in appendix $\mathrm{C}$ that all elements of $H_{\bar{\partial}_{B}}^{2,1}$ and $H_{\bar{\partial}_{B}}^{1,2}$ are primitive.

The multiplets corresponding to the groups $H_{\bar{\partial}_{B}}^{2,1}$ and $H_{\bar{\partial}_{B}}^{1,2}$ contribute differently to the index. Multiplets corresponding to $H_{\bar{\partial}_{B}}^{1,2}$ have a $(1,2)$ form as their primary and an accompanying Lichnerowicz mode. However the situation is reversed for multiplets corresponding to $H_{\bar{\partial}_{B}}^{2,1}$; their primary is a Lichnerowicz mode and they have an accompanying $(2,1)$ form. Thus, the two modes contribute differently to the index. Hypermultiplets appear in complex conjugate pairs. For each hypermultiplet containing a $(1,2)$ form, there is another hypermultiplet with a $(2,1)$ form of opposite charge. If a hypermultipet contributes to the index, its conjugate does not necessarily have to contribute as well. One has to be careful to avoid overcounting of modes with charge zero. We propose that the zero-charge sector should not contribute to either hypermultiplet, since the relevant three-forms carry zero $\mathrm{U}(1)$-charge and are thus closed. It is likely that they are gauge modes.

\section{Cohomology calculations}

In this section, we explain how to evaluate the trace over the cohomology groups contributing to the index. We consider both toric and Fano manifolds. We find several remarkable cancellations that simplify the index.

\subsection{Toric Calabi-Yau varieties}

Let us consider a toric Calabi-Yau cone $X$. In this case, there are one superconformal R-symmetry and three mesonic flavor symmetries. We will take a new basis of these symmetries such that the exponentiated chemical potentials are given by $x_{1}, x_{2}, x_{3}, x_{4}$ with $t^{2}=x_{1} x_{2} x_{3} x_{4}$. Then each holomorphic function $f$ has integer charges $\mathbf{q}=\left(q_{1}, q_{2}, q_{3}, q_{4}\right)$ under the four isometries, and contributes $\mathbf{x}^{\mathbf{q}}=x_{1}{ }^{q_{1}} x_{2}{ }^{q_{2}} x_{3}{ }^{q_{3}} x_{4}{ }^{q_{4}}$ to the index. The charges form a cone $M \subset \mathbb{Z}^{4}$ and

$$
\operatorname{Tr} \mathbf{x}^{\mathbf{q}} \mid H^{0}\left(X, \mathcal{O}_{X}\right)=\sum_{\mathbf{q} \in M} \mathbf{x}^{\mathbf{q}}
$$

\footnotetext{
${ }^{5}$ It would be interesting to find or exclude the possibility of additional charged modes.
} 
Since $\wedge^{3} \Omega_{X}^{\prime}$ carries R-charge two,

$$
\operatorname{Tr} \mathbf{x}^{\mathbf{q}} \mid H^{0}\left(X, \wedge^{3} \Omega_{X}^{\prime}\right)=\sum_{\mathbf{q} \in M} \mathbf{x}^{\mathbf{q}+(1,1,1,1)}
$$

The groups $H^{\geq 1}\left(X, \wedge{ }^{k} \Omega_{X}^{\prime}\right)$ vanish. The characters of the ordinary and reduced differential forms are

$$
\begin{aligned}
& \operatorname{Tr} \mathbf{x}^{\mathbf{q}} \mid H^{0}\left(X, \wedge^{k} \Omega_{X}\right)=\sum_{\mathbf{q} \in M} \tilde{n}_{\mathbf{q}}^{k} \mathbf{x}^{\mathbf{q}} \\
& \operatorname{Tr} \mathbf{x}^{\mathbf{q}} \mid H^{0}\left(X, \wedge^{k} \Omega_{X}^{\prime}\right)=\sum_{\mathbf{q} \in M} n_{\mathbf{q}}^{k} \mathbf{x}^{\mathbf{q}} .
\end{aligned}
$$

These characters are $[28,29]$

$$
\begin{aligned}
& \tilde{n}_{q}^{k}= \begin{cases}0 & \text { if } q \text { is on a vertex of } M, \\
\left(\begin{array}{c}
m \\
k
\end{array}\right) & \text { if } q \text { is on a } m \text {-dimensional facet of } M,\end{cases} \\
& n_{q}^{k}= \begin{cases}0 & \text { if } q \text { is on a vertex of } M, \\
\left(\begin{array}{c}
m-1 \\
k
\end{array}\right) & \text { if } q \text { is on a } m \text {-dimensional facet of } M .\end{cases}
\end{aligned}
$$

Let $M^{(m)}$ be the set of points of $M$ that are contained in a $m$-dimensional facet and let $M^{\circ(m)}$ be the set of points of $M$ that are contained in the interior of a $m$-dimensional facet. Since all of the points of $M$ are in the interior of some facet, we have

$$
\sum_{k=1}^{\operatorname{dim} X} \sum_{\mathbf{q} \in M^{\circ}(m)} \mathbf{x}^{\mathbf{q}}=\sum_{\mathbf{q} \in M^{(\operatorname{dim} X)}} \mathbf{x}^{\mathbf{q}}=\sum_{\mathbf{q} \in M^{\circ}(m)} \mathbf{x}^{\mathbf{q}+(1,1,1,1)} .
$$

Using these identities, we can write the index as

$$
1+\mathcal{I}_{\text {s.t. }}=-b_{2}(Y) \mathbf{x}^{(1,1,1,1)}+\sum_{\mathbf{q} \in M^{(2)}}\left(\mathbf{x}^{\mathbf{q}}-\mathbf{x}^{\mathbf{q}+(1,1,1,1)}\right)-\sum_{\mathbf{q} \in M^{(1)}} \mathbf{x}^{\mathbf{q}+(1,1,1,1)}
$$

Since we computed the cohomology of the singular toric variety, we missed the contribution of the of $b_{2}(Y)$ Betti multiplets which only appear on the resolved geometry, so we must add their contribution to the index.

\subsection{Toric examples}

We now illustrate our general formula for toric cohomologies in two simple examples. Our first example is the cone over $Q^{1,1,1}$. The R-symmetry group is $\mathrm{SU}(2)^{3} \times \mathrm{U}(1)_{R}$. The holomorphic functions of R-charge $t^{l}$ for integer $l$ are in one-to-one correspondence with the lattice points of the cube $[0, l]^{3}$. The flavor fugacity transforms in the $[l, l, l]$ representation of $\mathrm{SU}(2)^{3}$ of dimension $(l+1)^{3}$. The lattice points are shown in figure 1 for $l=3$. In table 3 we list the contributions to $M^{\circ}(m)$ for small $l$. Using this data, we the find the 


\begin{tabular}{|l|c|cccccc|}
\hline Contribution & Fugacity & 1 & $t$ & $t^{2}$ & $t^{3}$ & $t^{4}$ & $\ldots$ \\
\hline$\sum_{\mathbf{q} \in M^{(2)}}$ & $\mathbf{x}^{\mathbf{q}}$ & 1 & 8 & 20 & 32 & 44 & $\ldots$ \\
$\sum_{\mathbf{q} \in M^{(2)}}$ & $-\mathbf{x}^{\mathbf{q}+(1,1,1,1)}$ & 0 & 0 & 1 & 8 & 20 & $\ldots$ \\
$\sum_{\mathbf{q} \in M^{(1)}}$ & $-\mathbf{x}^{\mathbf{q}+(1,1,1,1)}$ & 0 & 0 & 1 & 8 & 8 & $\ldots$ \\
$b_{2}(Y)$ & $-\mathbf{x}^{(1,1,1,1)}$ & 0 & 0 & 2 & 0 & 0 & $\ldots$ \\
\hline $1+\mathcal{I}_{\text {s.t. }}$ & $(-1)^{F} \mathbf{x}^{\epsilon+j_{3}}$ & 1 & 8 & 16 & 16 & 16 & $\ldots$ \\
\hline
\end{tabular}

Table 2. Contributions to the index of $Q^{1,1,1}$.

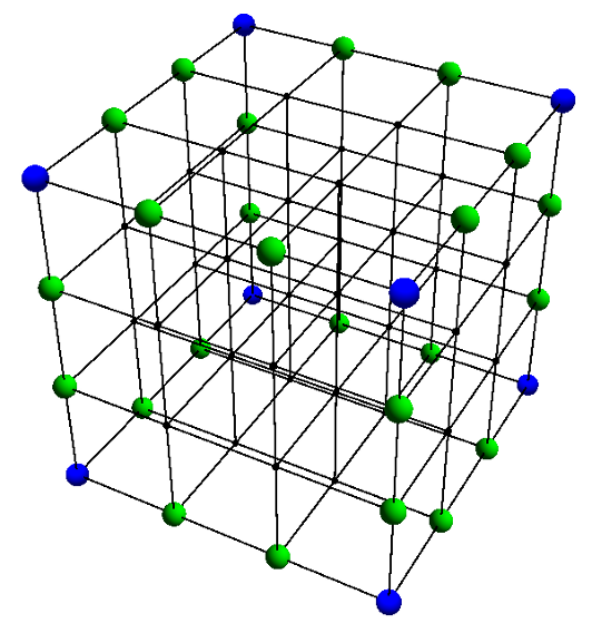

Figure 1. Contributions to $M^{\circ(1)}$ and $M^{\circ(2)}$ for $Q^{1,1,1}$ with $l=3$ are colored green and blue respectively.

\begin{tabular}{|c|cccc|}
\hline$l$ & $M^{\circ(1)}$ & $M^{\circ(2)}$ & $M^{\circ(3)}$ & $M^{\circ(4)}$ \\
\hline 0 & 1 & 0 & 0 & 0 \\
1 & 8 & 0 & 0 & 0 \\
2 & 8 & 12 & 6 & 1 \\
3 & 8 & 24 & 24 & 8 \\
4 & 8 & 36 & 54 & 27 \\
5 & 8 & 48 & 96 & 64 \\
\hline
\end{tabular}

Table 3. Toric data for $Q^{1,1,1}$. 


\begin{tabular}{|l|c|cccccccc|}
\hline Contribution & Fugacity & 1 & $t^{1 / 2}$ & $t$ & $t^{3 / 2}$ & $t^{2}$ & $t^{5 / 2}$ & $t^{3}$ & $\ldots$ \\
\hline$\sum_{\mathbf{q} \in M^{(2)}}$ & $\mathbf{x}^{\mathbf{q}}$ & 1 & 4 & 10 & 16 & 22 & 28 & 34 & $\ldots$ \\
$\sum_{\mathbf{q} \in M^{(2)}}$ & $-\mathbf{x}^{\mathbf{q}+(1,1,1,1)}$ & 0 & 0 & 0 & 0 & 1 & 4 & 10 & $\ldots$ \\
$\sum_{\mathbf{q} \in M^{(1)}}$ & $-\mathbf{x}^{\mathbf{q}+(1,1,1,1)}$ & 0 & 0 & 0 & 0 & 1 & 4 & 4 & $\ldots$ \\
$b_{2}(Y)$ & $-\mathbf{x}^{(1,1,1,1)}$ & 0 & 0 & 0 & 0 & 0 & 0 & 0 & $\ldots$ \\
\hline $1+\mathcal{I}_{\text {s.t. }}$ & $(-1)^{F} \mathbf{x}^{\epsilon+j_{3}}$ & 1 & 4 & 10 & 16 & 20 & 20 & 20 & $\ldots$ \\
\hline
\end{tabular}

Table 4. Contributions to the index of $S^{7}$.

contributions to the index listed in table 2. Summing all the contributions to the index, we find

$$
\mathcal{I}_{\text {s.t. }}\left(Q^{1,1,1} ; t\right)=8 t+\frac{16 t^{2}}{1-t} .
$$

Our second example is the seven-sphere $S^{7}$. The cone over $S^{7}$ is the four-dimensional complex space $\mathbb{C}^{4}$. The ring of holomorphic functions is $\mathbb{C}\left[x_{1}, x_{2}, x_{3}, x_{4}\right]$. Monomials with fixed degree $l$ have R-charge $l / 2$ and correspond to the lattice points of a tetrahedron with $(l+1)$ lattice points on each side. We list the contributions to the index in table 4 . Summing the contributions to the index, we find

$$
\mathcal{I}_{\text {s.t. }}\left(S^{7} ; t\right)=4 t^{1 / 2}+10 t+16 t^{3 / 2} \frac{20 t^{2}}{1-t^{1 / 2}} .
$$

\subsection{Cones over Fano varieties}

Our results take an especially nice form for cones over Fano threefolds. A smooth projective variety is called Fano if its anti-canonical divisor $-K_{V} \cong \operatorname{det} T_{V}$ is ample. We now introduce several invariants of Fano threefolds $V$ and express them in terms of the Chern classes $c_{i}(V)$. The degree of $V$ is $d=-K_{V}^{3}=\int_{V} c_{1}^{3}$ and the Euler characteristic $\chi(V)=\int_{V} c_{3}=$ $2\left(1+b_{2}-b_{3}\right)$. We will simplify many expressions using $\int_{V} c_{1}^{2} c_{2}=24{ }^{6}$

The index $\operatorname{ind}(V)$ is the largest integer $r$ such that there exists a divisor $H$ such that $r H \cong-K_{V}$. We call $H$ the fundamental divisor. Let $\mathcal{L}$ be the line bundle corresponding to the fundamental divisor. If $V$ has a Kähler-Einstein metric then the total space of the fibration $\mathcal{L} \rightarrow V$ is a Calabi-Yau cone. The unit circle bundle in $\mathcal{L}$ is a Sasaki-Einstein manifold $[24,30]$. For simplicity we will restrict our attention to this case.

Fano threefolds are classified by the work of Fano, Iskovskikh, Shokurov, Mori, and Mukai. The index of an n-dimensional Fano variety satisfies $\operatorname{ind}(V) \leq n+1$. The complex projective space $\mathbb{C P}^{3}$ is the unique Fano threefold with index 4 . Similarly the quadric hypersurface $Q$ in $\mathbb{C P}^{4}$ is the unique Fano threefold with index 3 . There are 105 deformation families of Fano threefolds and they are described in [31]. We list a few of the simplest Fano threefolds in table 5.

\footnotetext{
${ }^{6}$ This relation can be derived using the Hirzebruch-Riemann-Roch theorem to calculate $\chi\left(\mathcal{O}_{V}\right)=1$.
} 


\begin{tabular}{|c|c|c|c|c|c|}
\hline$S E_{7}$ & Fano & $b_{2}(Y)$ & $\chi(V)$ & ind $(V)$ & $-K_{V}^{3}$ \\
\hline$S^{7}$ & $\mathbb{C P}^{3}$ & 0 & 4 & 4 & 64 \\
$M^{1,1,1}$ & $\mathbb{C P}^{2} \times \mathbb{C P}^{1}$ & 1 & 6 & 1 & 54 \\
$Q^{1,1,1}$ & $\mathbb{C P}^{1} \times \mathbb{C P}^{1} \times \mathbb{C P}^{1}$ & 2 & 8 & 2 & 48 \\
$V_{5,2}$ & $Q$ & 0 & 4 & 3 & 54 \\
\hline
\end{tabular}

Table 5. Some Fano threefolds and their Sasaki-Einstein cones.

\subsection{Twisted cohomology of Fano varities}

In this section we compute the cohomology groups contributing to the superconformal index for cones over Fano threefolds. Since $V$ is Fano, its anti-canonical divisor $-K_{V}$ is ample and the cohomology groups $H^{i \geq 1}\left(V, \mathcal{L}^{j}\right)$ vanish by the Kodaira vanishing theorem. The first contribution to the index is the Hilbert series. The Hilbert series of the cone can be computed using the Hirzebruch-Riemann-Roch theorem since all higher cohomology groups vanish. We compute the Hilbert series using

$$
\begin{aligned}
C(t, V) & =\sum_{j \geq 0} t^{2 j / r} \chi\left(V, \mathcal{L}^{j}\right) \\
& =\sum_{j \geq 0} t^{2 j / r} \int e^{-j c_{1}(\mathcal{L})} \cdot \operatorname{Todd}(V),
\end{aligned}
$$

where $r$ is the Fano-index. The Todd class can be expressed in terms of the Chern classes of the tangent bundle $T V$ as

$$
T d(V)=1+\frac{c_{1}(T V)}{2}+\frac{c_{1}(T V)^{2}+c_{2}(T V)}{12}+\frac{c_{1}(T V) c_{2}(T V)}{24} .
$$

For Fano threefolds of index $1, \mathcal{L}=-K_{V}$ and

$$
e^{-c_{1}(\mathcal{L})}=1+c_{1}\left(K_{V}\right)+c_{1}\left(K_{V}\right)^{2} / 2+\ldots
$$

Combining these ingredients, we find that the Hilbert series is

$$
C(t, V)=\frac{1+(d / 2-1) t^{2}+(d / 2-1) t^{4}+t^{6}}{\left(1-t^{2}\right)^{4}}
$$

where $d$ is the degree. We can similarly compute the other contributions to the superconformal index using the Hirzebruch-Riemann-Roch theorem. Starting with our general formula for the index in equation (2.3), we can organize the contributions to the index in terms of the characters

$$
C^{j}(t, V)=\sum_{n} t^{n} \chi\left(V, \wedge^{j} \Omega_{V} \otimes \mathcal{L}^{n}\right)
$$

Using Kodaira-Nakano vanishing theorem, $H^{p}\left(V, \wedge^{q} \Omega_{V} \otimes \mathcal{L}^{n}\right)=0$ for $p+q>\operatorname{dim}(V)$, we find that the superconformal index is

$$
1+\mathcal{I}_{\text {s.t. }}(t)=C^{0}-C^{2}+t^{2}\left(C^{1}-C^{3}\right) .
$$




\begin{tabular}{|l|c|l|l|l|c|}
\hline Multiplet & $j_{3}$ & Primary $y$ & Multiplicity & $\mathrm{SU}(4)_{\mathrm{U}(1)_{R}}$ & Index \\
\hline short graviton & 1 & $\ell / 2-2$ & $H^{0}\left(X, \wedge^{3} \Omega_{X}^{\prime}\right)$ & {$[\ell-4,0,0]_{\ell / 2}$} & $-t^{\ell / 2+2}$ \\
short gravitino & $1 / 2$ & $\ell / 2-1$ & $H^{0}\left(X, \Omega_{X}^{\prime}\right)$ & {$[\ell-2,1,0]_{\ell / 2}$} & $t^{\ell / 2+2}$ \\
short vector $A$ & 0 & $\ell / 2-2$ & $H^{0}\left(X, \wedge^{2} \Omega_{X}^{\prime}\right)$ & {$[\ell-3,0,1]_{\ell / 2}$} & $-t^{\ell / 2}$ \\
hyper & 0 & $\ell / 2$ & $H^{0}\left(X, \mathcal{O}_{X}\right)$ & {$[\ell, 0,0]_{\ell / 2}$} & $t^{\ell / 2}$ \\
\hline
\end{tabular}

Table 6. Short multiplets for $A d S_{4} \times S^{7}$ and their contribution to the index. Some short multiplets can become massless for small $\ell$.

For Fano threefolds of index $\operatorname{ind}(V)=1, \mathcal{L}=-K_{V}$, and we find that the superconformal index is

$$
\mathcal{I}_{\text {s.t. }}(t)=\frac{(24-\chi(V)) t^{2}}{1-t^{2}} .
$$

For Fano threefolds of index ind $(V)=2$ :

$$
\mathcal{I}_{\text {s.t. }}(t)=(12-\chi(V) / 2) t+\frac{(24-\chi(V)) t^{2}}{1-t} .
$$

We can also compute the index for $\mathbb{C P}^{3}$ and the quadric $Q$ with all flavor fugacities set to one. We find that the superconformal indices are

$$
\begin{aligned}
& \mathcal{I}_{\text {s.t. }}\left(\mathbb{C P}^{3} ; t\right)=4 t^{1 / 2}+10 t+16 t^{3 / 2}+\frac{20 t^{2}}{1-t^{1 / 2}}, \text { and } \\
& \mathcal{I}_{\text {s.t. }}(Q ; t)=6 t^{2 / 3}+14 t^{4 / 3}+\frac{20 t^{2}}{1-t^{2 / 3}} .
\end{aligned}
$$

In the next section we will derive the superconformal indices for the cones over $\mathbb{C P}^{3}$ and the quadric, $Q$, refined by their flavor fugacities.

\subsection{The seven-sphere $S^{7}$}

The seven-sphere $S^{7}$ is the unit circle bundle over $\mathbb{C P}^{3}$. Its isometry group is $\mathrm{SU}(4) \times \mathrm{U}(1)_{R}$. Using the twisted cohomology groups of complex projective space listed in appendix D, we find the short multiplets in supergravity and list them in table 6. Summing all contributions to the index, we find

$$
1+\mathcal{I}_{\text {s.t. }}\left(S^{7} ; t\right)=\sum_{n \geq 0}\left(1-t^{4}\right) t^{n / 2} \chi_{[n, 0,0]}^{\mathrm{SU}(4)}-t^{n / 2+3 / 2} \chi_{[n, 0,1]}^{\mathrm{SU}(4)}+t^{n / 2+3} \chi_{[n, 1,0]}^{\mathrm{SU}(4)} \text {. }
$$

This is in complete agreement with the original derivation of the supergravity index [4] and the field theory index $[6,7]$.

\subsection{The Stiefel manifold $V_{5,2}$}

The Stiefel manifold $V_{5,2}=\mathrm{SO}(5) / \mathrm{SO}(3)$ is the unit circle bundle over the quadric $Q \in \mathbb{C P}^{4}$. Its isometry group is $\mathrm{SO}(5) \times \mathrm{U}(1)_{R}$. Using the twisted cohomology groups of the quadric 


\begin{tabular}{|l|c|l|l|l|c|}
\hline Multiplet & $j_{3}$ & Primary $y$ & Multiplicity & $\mathrm{SO}(5)_{\mathrm{U}(1)_{R}}$ & Index \\
\hline short graviton & 1 & $2 \ell / 3-2$ & $H^{0}\left(X, \wedge^{3} \Omega_{X}^{\prime}\right)$ & {$[\ell-3]_{2 \ell / 3}$} & $-t^{2 \ell / 3+2}$ \\
short gravitino & $1 / 2$ & $2 \ell / 3-1$ & $H^{0}\left(X, \Omega_{X}^{\prime}\right)$ & {$[\ell-2,2]_{2 \ell / 3}$} & $t^{2 \ell / 3+2}$ \\
short vector $A$ & 0 & $2 \ell / 3-2$ & $H^{0}\left(X, \wedge^{2} \Omega_{X}^{\prime}\right)$ & {$[\ell-3,2]_{2 \ell / 3}$} & $-t^{2 \ell / 3}$ \\
hyper & 0 & $2 \ell / 3$ & $H^{0}\left(X, \mathcal{O}_{X}\right)$ & {$[\ell, 0]_{2 \ell / 3}$} & $t^{2 \ell / 3}$ \\
hyper & 0 & $2 \ell / 3$ & $H^{1}\left(X, \wedge^{2} \Omega_{X}^{\prime}\right)$ & {$[0,0]_{2 / 3}$} & $t^{2 / 3}$ \\
\hline
\end{tabular}

Table 7. Short multiplets for $V_{5,2}$. Here the $\mathrm{U}(1)_{R}$ charge is $y$.

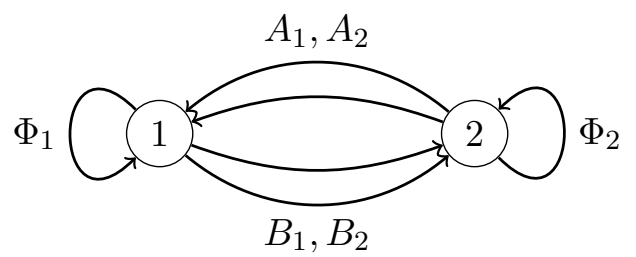

Figure 2. Quiver for $V_{5,2}$.

listed in appendix D, we find the short multiplets in supergravity and list them in table 7. We write $\mathrm{SO}(5)$ representations in terms of the Dynkin labels $\left[\lambda_{1}, \lambda_{2}\right]$ of their highest

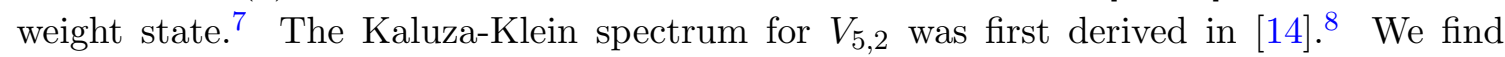
that one of the short gravitino multiplets listed in [14] is extraneous. The non-trivial cohomology group $H^{1}\left(Q, \Omega^{2}(1)\right) \cong \mathbb{C}$ implies the existence of a short hypermultiplet also missing from [14]. This hypermultiplet and its anti-holomorphic partner were previously found in [33]. Summing all contributions, the gravity superconformal index is

$$
1+\mathcal{I}_{\text {s.t. }}\left(V_{5,2} ; t\right)=t^{2 / 3}+\sum_{n \geq 0}\left(1-t^{4}\right) t^{2 / 3 n} \chi_{[n, 0]}^{\mathrm{SO}(5)}-\left(1-t^{4 / 3}\right) \chi_{[n, 2]}^{\mathrm{SO}(5)} t^{2+2 / 3 n},
$$

which refines the index in equation (3.22) by the $\mathrm{SO}(5)$ flavor fugacities.

The field theory dual to M2-branes at the cone over $V_{5,2}$ was recently proposed to be a $\mathrm{U}(N) \times \mathrm{U}(N)$ gauge theory with Chern-Simons interactions [34]. The bi-fundamental and adjoint matter content is shown as a quiver in figure 2. The manifest global symmetry is $\mathrm{SU}(2) \times \mathrm{U}(1)_{B} \times \mathrm{U}(1)_{R}$. The pairs of bifundamental fields $\left(A_{1}, A_{2}\right)$ and $\left(B_{1}, B_{2}\right)$ transform as doublets under the global $\mathrm{SU}(2)$ symmetry. The field theory index was computed in [12] up to terms of order $t^{2}$. The gravity index we compute in equation (3.24) exactly matches the known field theory terms. However, a few more terms are desirable, since the leading contribution of the short gravitino multiplet in [14] is at order $t^{10 / 3}$. While it is possible to directly extend the field theory computation to arbitrary fixed order, we instead compute the index to all orders in $t$ in the zero-monopole sector for simplicity. Since the each pair of bi-fundamental fields transforms as a doublet under the $\mathrm{SU}(2)$ global symmetry, we assign

\footnotetext{
${ }^{7}$ If $\lambda_{2}$ is odd, the representation $\left[\lambda_{1}, \lambda_{2}\right]$ is spinor. If $\lambda_{2}$ is even, the representation is tensor and the corresponding Young tableaux has $\lambda_{1}$ height one columns and $\lambda_{2} / 2$ height two columns.

${ }^{8}$ Additional long multiplets were found in [32].
} 
the flavor fugacity $\chi_{1 / 2}^{\mathrm{SU}(2)}(z)=z^{1 / 2}+z^{-1 / 2}$ to each pair. Let $M_{Q}(t, z)$ be the adjacency matrix of the $V_{5,2}$ quiver weighted by the R-charges and flavor fugacities,

$$
M_{Q}(t, z)=\left(\begin{array}{cc}
t^{2 / 3} & t^{2 / 3} \chi_{1 / 2}^{\mathrm{SU}(2)}(z) \\
t^{2 / 3} \chi_{1 / 2}^{\mathrm{SU}(2)}(z) & t^{2 / 3}
\end{array}\right),
$$

and let $\chi_{Q}(t, z)$ be the matrix constructed from the single-letter indices. In terms of the adjacency matrix, $\chi_{Q}(t, z)$ has the following simple form,

$$
\chi_{Q}(t, z)=\frac{1}{\left(1-t^{2}\right)}\left(1-M_{Q}(t, z)+t^{2} M_{Q}^{T}\left(t^{-1}, z^{-1}\right)-t^{2}\right) .
$$

The multi-trace contribution to the index from the letters is

$$
I^{(0)}=\prod_{n \geq 1} \frac{1}{\operatorname{det}\left(\chi_{Q}\left(t^{n}, z^{n}\right)\right)} .
$$

Using the plethystic logarithm we can extract the single-trace index in the zero-monopole sector

$$
\mathcal{I}_{\text {s.t. }}^{(0)}=\left(1-t^{2}\right) P E^{-1}\left[I^{(0)}\right]
$$

We find that the single-trace index in the zero-monopole sector is

$$
\mathcal{I}_{s . t .}^{(0)}=2 t^{2 / 3}+\left(2+z+z^{-1}\right) t^{4 / 3}+\sum_{n \geq 2}\left(z^{n}+z^{-n}\right)\left(1-t^{2 / 3}\right) t^{4 / 3 n}
$$

In supergravity, the zero-monopole sector corresponds to the states that remain in the orbifold $V_{5,2} / \mathbb{Z}_{k}$ in the large $k$ limit. Equivalently, these are the states that are singlets under $\mathrm{SU}(2)_{L}$ in the decomposition $\mathrm{SO}(5) \rightarrow \mathrm{SU}(2)_{L} \times \mathrm{SU}(2)_{R}$. Using the branching rules for $\mathrm{SO}(5)$, we verify that the supergravity index (3.24) exactly matches the field theory index in the zero-monopole sector.

\section{The supergravity analysis}

In this section we turn to the analysis of eleven-dimensional supergravity on $A d S_{4} \times S E_{7}$ manifolds. Standard results in gauge/string duality relate the operator spectrum of the dual gauge theory to the spectrum of various differential operators on the Sasaki-Einstein manifold. For reference, we give this dictionary in tables 20 and 21.

In section 4.1 we determine the spectrum of $\Delta$ and $Q=\star d$. We leave the analysis of fermionic and Lichnerowicz eigenmodes to a future work. Section 4.2 explains how to arrange the various modes into multiplets.

\subsection{The Kaluza-Klein spectrum}

The Kaluza-Klein spectra of various coset manifolds have been successfully studied using harmonic analysis. For more general manifolds however, the problem becomes considerably more difficult. Hence as in [13] our strategy is to use less information; i.e. we start with an eigenform of one of the differential operators and then use the Sasaki-Einstein structure to 


\begin{tabular}{|c|c|c|c|c|}
\hline Name & Degree & $\Delta, Q$ & Charge & \\
\hline$f^{[0 ; q]}$ & 0 & $\delta$ & $q$ & $c, \star, \bar{\star}$ \\
\hline$d f^{[0 ; q]}$ & 1 & $\delta$ & $q$ & \\
\hline$f^{[1 ; q ;-]}$ & 1 & $\delta+6-2 \sqrt{\delta+9}$ & $q$ & $\bar{\star}$ \\
\hline$f^{[1 ; q ;+]}$ & 1 & $\delta+6+2 \sqrt{\delta+9}$ & $q$ & $c, \star$ \\
\hline$f^{[2 ; q ; a]}$ & 2 & $\delta+8$ & $q$ & $\star, \bar{\star}$ \\
\hline$f^{[2 ; q ; b]}$ & 2 & $\delta+8$ & $q$ & \\
\hline$d f^{[1 ; q ;-]}$ & 2 & $\delta+6-2 \sqrt{\delta+9}$ & $q$ & \\
\hline$d f^{[1 ; q ;+]}$ & 2 & $\delta+6+2 \sqrt{\delta+9}$ & $q$ & \\
\hline$f^{[2 ; q+4]}$ & 2 & $\delta+8$ & $q+4$ & $\bar{\star}$ \\
\hline$f^{[2 ; q-4]}$ & 2 & $\delta+8$ & $q-4$ & $\star$ \\
\hline$f^{[3 ; q ;-]}$ & 3 & $1-\sqrt{\delta+9}$ & $q$ & ॠ \\
\hline$f^{[3 ; q ;+]}$ & 3 & $1+\sqrt{\delta+9}$ & $q$ & $c, \star$ \\
\hline$f^{[3 ; q+4 ;-]}$ & 3 & $1-\sqrt{\delta+9}$ & $q+4$ & ॠ \\
\hline$f^{[3 ; q+4 ;+]}$ & 3 & $1+\sqrt{\delta+9}$ & $q+4$ & $c, \star, \bar{\star}$ \\
\hline$f^{[3 ; q-4 ;-]}$ & 3 & $1-\sqrt{\delta+9}$ & $q-4$ & $\star, \bar{\star}$ \\
\hline$f^{[3 ; q-4 ;+]}$ & 3 & $1+\sqrt{\delta+9}$ & $q-4$ & $c, \star$ \\
\hline
\end{tabular}

Table 8. Wave functions $f^{[p ; q ; X]}$ derived from a scalar $f . p$ refers to the form degree, $q$ to its charge, $X$ denotes any additional labels. Non-gauge modes that remain for holomorphic $f$-i.e. $\bar{\partial}_{B} f=0$ - are labeled with " $\star$ ". " " " marks the anti-holomorphic case. The modes with constant $f$ are labeled " $c$ ". Neither of $f^{[2 ; q ; a, b]}$ vanishes when $f$ is (anti-) holomorphic, yet they coincide. We label this mode $f^{[2 ; q ; a]}$.

construct additional eigenforms. In other words, given an eigenfunction $f$ with $\Delta f=\delta f$, we use interior and exterior products with the forms and operators $\eta, J, \Omega, \partial_{B}, \bar{\partial}_{B}, £_{\xi}$. We then identify the short multiplets with the Kohn-Rossi cohomology groups $H_{\bar{\partial}_{B}}^{p, q}$ and the related groups $H^{q}\left(X, \wedge^{p} \Omega_{X}^{\prime}\right)$ on the cone. Our conventions concerning Sasaki-Einstein manifolds, Kohn-Rossi cohomology, and the various differential operators are listed in appendix A. For details of the analysis the reader should refer to appendix B. An analysis of the compatibility of Lefschetz decomposition with Kohn-Rossi cohomology is performed in appendix $\mathrm{C}$.

Eigenmodes constructed from scalars $f,(1,0)$ forms $\sigma$, primitive $(1,1)$ forms $\chi$, and primitive $(2,1)$ forms $\zeta$ are listed in tables $8,9,10$, and 11 . Various conditions - most noteably primitivity - simply stem from the fact that we impose orthogonality between the various modes in order to avoid overcounting. Since the basis we use for the scalars 


\begin{tabular}{|ccccc|}
\hline Name & Degree & $\Delta, Q$ & Charge & \\
\hline$\sigma^{[1 ; q]}$ & 1 & $\delta$ & $q$ & $\star$ \\
$\sigma^{[1 ; q-4]}$ & 1 & $\delta$ & $q-4$ & $\star$ \\
\hline$\sigma^{[2 ; q ;-]}$ & 2 & $\delta+4-2 \sqrt{\delta+4}$ & $q$ & \\
$\sigma^{[2 ; q ;+]}$ & 2 & $\delta+4+2 \sqrt{\delta+4}$ & $q$ & $\star$ \\
$\sigma^{[2 ; q-4 ;-]}$ & 2 & $\delta+4-2 \sqrt{\delta+4}$ & $q-4$ & $\star$ \\
$\sigma^{[2 ; q-4 ;+]}$ & 2 & $\delta+4+2 \sqrt{\delta+4}$ & $q-4$ & $\star$ \\
\hline$\sigma^{[3 ; q ;-]}$ & 3 & $-\sqrt{\delta+4}$ & $q$ & \\
$\sigma^{[3 ; q ;+]}$ & 3 & $\sqrt{\delta+4}$ & $q$ & $\star$ \\
$\sigma^{[3 ; q-4 ;-]}$ & 3 & $-\sqrt{\delta+4}$ & $q-4$ & $\star$ \\
$\sigma^{[3 ; q-4 ;+]}$ & 3 & $\sqrt{\delta+4}$ & $q-4$ & $\star$ \\
\hline
\end{tabular}

Table 9. Wave functions $\sigma^{[p ; q ; X]}$ derived from a $(1,0)$-form $\sigma$. Again, holomorphic modes are marked "夫".

\begin{tabular}{|ccccc|}
\hline Name & Degree & $\Delta, Q$ & Charge & \\
\hline$\chi^{[2 ; q]}$ & 2 & $\delta$ & $q$ & $\star, \square$ \\
\hline$\chi^{[3 ; q ;-]}$ & 3 & $-1-\sqrt{\delta+1}$ & $q$ & $\star, \square$ \\
$\chi^{[3 ; q ;+]}$ & 3 & $-1+\sqrt{\delta+1}$ & $q$ & \\
\hline
\end{tabular}

Table 10. Wave functions $\chi^{[p ; q ; X]}$ derived from a primitive $(1,1)$-form $\chi$. "^" marks the modes remaining when $\chi$ is holomorphic and $\delta=q(q+2)$, " $\square$ " those with $d \chi=0$ and $\delta=q=0$.

\begin{tabular}{|ccccc|}
\hline Name & Degree & $Q$ & Charge & \\
\hline$\zeta^{[3 ; q]}$ & 3 & $-q$ & $q$ & $\star$ \\
$\vartheta^{[3 ; q]}$ & 3 & $q$ & $q$ & $\star$ \\
\hline
\end{tabular}

Table 11. Wave functions based on three-forms.

includes $f \eta, f J, f \eta \wedge J$, the higher forms have to be orthogonal to both the Reeb vector and the Kähler form. Further conditions are discussed in the appendix.

A crucial role is played by Kohn-Rossi holomorphic forms. Whenever forms or scalars are holomorphic (i.e. annihilated by $\bar{\partial}_{B}$ ), a number of derived modes vanish. This will lead to multiplet shortening. The surviving modes are marked in the tables. Moreover, given a function $f$ and $£_{\xi} f=\imath q f$, one can show that the Laplace operator is bounded 
(see eq. (B.15))

$$
\Delta f \geq q(q+6) f
$$

Equality holds if and only if $f$ is holomorphic. Our calculations in appendix B suggest that the suitable generalization for primitive $k$-forms orthogonal to the Reeb vector is $(k=0,1,2,3)$

$$
\Delta_{k} \geq q[q+(6-2 k)]=4 y_{\bar{\partial}_{B}}\left[y_{\bar{\partial}_{B}}+(3-k)\right] .
$$

Here $y_{\bar{\partial}_{B}}=2 q$. Again, equality holds if and only if the form is holomorphic. This inequality prompts us to define $E_{\bar{\partial}_{B}}$ via

$$
\Delta_{k}=4 E_{\bar{\partial}_{B}}\left[E_{\bar{\partial}_{B}}+(3-k)\right]
$$

In these variables, the bound becomes

$$
E_{\bar{\partial}_{B}} \geq y_{\bar{\partial}_{B}} \cdot
$$

It would be very interesting to find a further generalization of (4.2) that holds for all $k$-forms. The minimal modification that satisfies $\Delta J=12 J$ and $\Delta \eta=12 \eta$ is

$$
\left.\left.\Delta=2 \Delta_{\bar{\partial}_{B}}-£_{\xi}^{2}-2 \imath(3-k) £_{\xi}+2 J \wedge J\right\lrcorner+6 \eta \wedge \eta\right\lrcorner .
$$

$\Delta_{\bar{\partial}_{B}}$ is the Kohn-Rossi Laplacian, $\Delta_{\bar{\partial}_{B}}=\bar{\partial}_{B}^{\dagger} \bar{\partial}_{B}+\bar{\partial}_{B} \bar{\partial}_{B}^{\dagger}$. In this form (4.5) begins to resemble the well-known identity for the de Rham and the Dolbeault Laplacians on Kähler manifolds, $\Delta=2 \Delta_{\partial}=2 \Delta_{\bar{\partial}}$.

\subsection{The multiplets}

In this section, we will arrange the Kaluza-Klein modes into superconformal multiplets. Substituting the Kaluza-Klein modes derived in section 4.1 into tables 20 and 21 yields the states of the dual SCFT. The superconformal primary has energy, spin, and hypercharge $\left(E_{0}, j_{3}, y\right)$. These variables differ from $E_{\bar{\partial}_{B}}$ and $y_{\bar{\partial}_{B}}$ by constant shifts. The superconformal primaries are labeled " $p$ ". Our analysis here has drawn on previous results obtained in the special case of coset manifolds. See e.g. [15, 35], and especially [16]. These references also include the fermionic modes. Wave-functions for which we have not derived an explicit expression are labeled with a subscript "*".

Long multiplets. The modes derived from a scalar $f$ in table 8 yield the graviton and vector multiplets $A$ and $W$ in tables 12,13 , and 14 . The third family of vector multiplets, the vector multiplet $Z$ (table 15 ) is based on the primitive $(1,1)$ forms of table 10 . Modes based on one-forms (see table 9) fill out the $\chi^{+}$and $\chi^{-}$gravitino multiplets (tables 16 and 17). The appearance of $f$ in the short graviton multiplet and long vector $W$ is known as the "shadow-mechanism" [36]. 


\begin{tabular}{|c|c|c|c|c|c|c|}
\hline Spin & Energy & Charge & Mass $^{2}$ & Name & Wave-f. & \\
\hline 2 & $E_{0}+1$ & $y$ & $4\left(E_{0}-2\right)\left(E_{0}+1\right)$ & $h$ & $f^{[0 ; q]}$ & $c, \star$ \\
$\frac{3}{2}$ & $E_{0}+\frac{1}{2}$ & $y+1$ & $E_{0}-2$ & $\chi^{+}$ & $f_{*}^{[3 / 2]}$ & $c, \star$ \\
1 & $E_{0}+2$ & $y$ & $4 E_{0}\left(E_{0}+1\right)$ & $W$ & $f^{[1 ; q ;-]}$ & \\
1 & $E_{0}+1$ & $y-2$ & $4 E_{0}\left(E_{0}-1\right)$ & $Z$ & $f^{[2 ; q-4]}$ & $\star$ \\
1 & $E_{0}+1$ & $y+2$ & $4 E_{0}\left(E_{0}-1\right)$ & $Z$ & $f^{[2 ; q+4]}$ & \\
1 & $E_{0}+1$ & $y$ & $4 E_{0}\left(E_{0}-1\right)$ & $Z$ & $f^{[2 ; q ; a, b]}$ & $\star$ \\
1 & $E_{0}+1$ & $y$ & $4 E_{0}\left(E_{0}-1\right)$ & $Z$ & $f^{[2 ; q ; a, b]}$ & \\
1 & $E_{0}$ & $y$ & $4\left(E_{0}-2\right)\left(E_{0}-1\right)$ & $A$ & $f^{[1 ; q ;+]}$ & $c, \star, p$ \\
0 & $E_{0}+1$ & $y$ & $4 E_{0}\left(E_{0}-1\right)$ & $\phi$ & $f_{*}^{\left[2_{s} ; q\right]}$ & \\
\hline
\end{tabular}

Table 12. The graviton multiplet. Modes surviving in the case that $f$ is holomorphic are labeled " $\star$ ". Then $E_{0}=y+2$. Moreover, modes remaining if $\mathrm{f}$ is constant are labeled with " $c$ " and satisfy $y=0$. They fill out the massless graviton multiplet. The normalization is such that $E_{\bar{\partial}_{B}}+2=E_{0}$ and $y_{\bar{\partial}_{B}}=y$.

\begin{tabular}{|c|c|c|c|c|c|c|}
\hline Spin & Energy & Charge & Mass $^{2}$ & Name & Wave-f. & \\
\hline 1 & $E_{0}+1$ & $y$ & $4 E_{0}\left(E_{0}-1\right)$ & $A$ & $f^{[1 ; q ;-]}$ & $m, \diamond$ \\
$\frac{1}{2}$ & $E_{0}+\frac{1}{2}$ & $y+1$ & $E_{0}-1$ & $\lambda_{L}$ & $f_{*}^{[1 / 2]}$ & $\diamond$ \\
0 & $E_{0}+2$ & $y$ & $4 E_{0}\left(E_{0}+1\right)$ & $\phi$ & $f_{*}^{[2 ; ; q]}$ & \\
0 & $E_{0}+1$ & $y-2$ & $4 E_{0}\left(E_{0}-1\right)$ & $\pi$ & $f^{[3 ; q-4 ;-]}$ & $\diamond$ \\
0 & $E_{0}+1$ & $y+2$ & $4 E_{0}\left(E_{0}-1\right)$ & $\pi$ & $f^{[3 ; q+4 ;-]}$ & \\
0 & $E_{0}+1$ & $y$ & $4 E_{0}\left(E_{0}-1\right)$ & $\pi$ & $f^{[3 ; q ;-]}$ & $m, \diamond$ \\
0 & $E_{0}$ & $y$ & $4\left(E_{0}-2\right)\left(E_{0}-1\right)$ & $S$ & $f^{[0 ; q]}$ & $m, \diamond, p$ \\
\hline
\end{tabular}

Table 13. The vector multiplet $A$. " $\diamond$ " marks the modes remaining when $\left.\bar{\partial}_{B} f\right\lrcorner \Omega$ defines a holomorphic $(2,0)$ form and the multiplet shortens. In this case $E_{0}=y+1$. The normalization is simple: $E_{\bar{\partial}_{B}}=E_{0}, y_{\bar{\partial}_{B}}=y$. " $m$ " marks the massless multiplet. We include the fermion mode contributing to the index.

\begin{tabular}{|c|c|c|c|c|c|c|}
\hline Spin & Energy & Charge & Mass $^{2}$ & Name & Wave-f. & \\
\hline 1 & $E_{0}+1$ & $y$ & $4 E_{0}\left(E_{0}-1\right)$ & $W$ & $f^{[1 ; q ;+]}$ & \\
0 & $E_{0}+2$ & $y$ & $4 E_{0}\left(E_{0}+1\right)$ & $\Sigma$ & $f^{[0 ; q]}$ & \\
0 & $E_{0}+1$ & $y-2$ & $4 E_{0}\left(E_{0}-1\right)$ & $\pi$ & $f^{[3 ; q-4 ;+]}$ & \\
0 & $E_{0}+1$ & $y+2$ & $4 E_{0}\left(E_{0}-1\right)$ & $\pi$ & $f^{[3 ; q+4 ;+]}$ & \\
0 & $E_{0}+1$ & $y$ & $4 E_{0}\left(E_{0}-1\right)$ & $\pi$ & $f^{[3 ; q ;+]}$ & \\
0 & $E_{0}$ & $y$ & $4\left(E_{0}-1\right)\left(E_{0}-2\right)$ & $\phi$ & $f_{*}^{[2 ; ; q]}$ & $p$ \\
\hline
\end{tabular}

Table 14. The vector multiplet $W$. The normalization is $E_{\bar{\partial}_{B}}+4=E_{0}, y_{\bar{\partial}_{B}}=y$. 


\begin{tabular}{|c|c|c|c|c|c|c|}
\hline Spin & Energy & Charge & Mass $^{2}$ & Name & Wave-f. & \\
\hline 1 & $E_{0}+1$ & $y$ & $4 E_{0}\left(E_{0}-1\right)$ & $Z$ & $\chi^{[2 ; q]}$ & $\star, \square$ \\
$\frac{1}{2}$ & $E_{0}+\frac{1}{2}$ & $y+1$ & $-E_{0}+1$ & $\lambda_{T}$ & $\chi_{*}^{[1 / 2]}$ & $\star, \square$ \\
0 & $E_{0}+2$ & $y$ & $4 E_{0}\left(E_{0}+1\right)$ & $\pi$ & $\chi^{[3 ; q ;+]}$ & \\
0 & $E_{0}+1$ & $y-2$ & $4 E_{0}\left(E_{0}-1\right)$ & $\phi$ & $\bar{\Omega} \cdot \chi_{*}^{[3 ; q ;-]}$ & $\star$ \\
0 & $E_{0}+1$ & $y+2$ & $4 E_{0}\left(E_{0}-1\right)$ & $\phi$ & $\Omega \cdot \chi_{*}^{[3 ; q ;+]}$ & \\
0 & $E_{0}+1$ & $y$ & $4 E_{0}\left(E_{0}-1\right)$ & $\phi$ & $J \cdot \chi_{*}^{[2 ; q]}$ & $\star, \square$ \\
0 & $E_{0}$ & $y$ & $4\left(E_{0}-1\right)\left(E_{0}-2\right)$ & $\pi$ & $\chi^{[3 ; q ;-]}$ & $\star, \square, p$ \\
\hline
\end{tabular}

Table 15. The vector multiplet $Z$. " $\star$ " marks modes remaining when $\chi$ is holomorphic. In this case $E_{0}=y+1$. The Betti multiplet is marked " $\square$ ". It corresponds to $d \chi=0$ and thus $y=0$. The Lichnerowicz mode vanishes since there is no $(2,1)$ form to contract $\bar{\Omega}$ with. The normalization is $E_{\bar{\partial}_{B}}+1=E_{0}, y_{\bar{\partial}_{B}}=y$.

\begin{tabular}{|c|c|c|c|c|c|c|}
\hline Spin & Energy & Charge & Mass $^{2}$ & Name & Wave-f. & \\
\hline 1 & $E_{0}+\frac{3}{2}$ & $y-1$ & $4\left(E_{0}-\frac{1}{2}\right)\left(E_{0}+\frac{1}{2}\right)$ & $Z$ & $\sigma^{[2 ; q-4 ;-]}$ & $\star$ \\
1 & $E_{0}+\frac{3}{2}$ & $y+1$ & $4\left(E_{0}-\frac{1}{2}\right)\left(E_{0}+\frac{1}{2}\right)$ & $Z$ & $\sigma^{[2 ; q ;-]}$ & \\
1 & $E_{0}+\frac{1}{2}$ & $y-1$ & $4\left(E_{0}-\frac{3}{2}\right)\left(E_{0}-\frac{1}{2}\right)$ & $A$ & $\sigma^{[1 ; q-4]}$ & $\star$ \\
1 & $E_{0}+\frac{1}{2}$ & $y+1$ & $4\left(E_{0}-\frac{3}{2}\right)\left(E_{0}-\frac{1}{2}\right)$ & $A$ & $\sigma^{[1 ; q]}$ & $\star$ \\
$\frac{1}{2}$ & $E_{0}$ & $y$ & $E_{0}-\frac{3}{2}$ & $\lambda_{L}$ & $\sigma_{*}^{[1 / 2]}$ & $\star$ \\
0 & $E_{0}+\frac{3}{2}$ & $y-1$ & $4\left(E_{0}-\frac{1}{2}\right)\left(E_{0}+\frac{1}{2}\right)$ & $\phi$ & $\sigma_{*}^{[2 ; q-4]}$ & $\star^{a}$ \\
0 & $E_{0}+\frac{3}{2}$ & $y+1$ & $4\left(E_{0}-\frac{1}{2}\right)\left(E_{0}+\frac{1}{2}\right)$ & $\phi$ & $\sigma_{*}^{\left[2_{s} ; q\right]}$ & $\star^{a}$ \\
0 & $E_{0}+\frac{1}{2}$ & $y-1$ & $4\left(E_{0}-\frac{3}{2}\right)\left(E_{0}-\frac{1}{2}\right)$ & $\pi$ & $\sigma^{[3 ; q-4 ;-]}$ & \\
0 & $E_{0}+\frac{1}{2}$ & $y+1$ & $4\left(E_{0}-\frac{3}{2}\right)\left(E_{0}-\frac{1}{2}\right)$ & $\pi$ & $\sigma^{[3 ; q ;-]}$ & \\
\hline
\end{tabular}

Table 16. The gravitino multiplet $\chi^{+}$is constructed from $(1,0)$ forms. Holomorphy yields the short gravitino multiplet, the relevant modes are marked " $\star$ ". Once again, $E_{\bar{\partial}_{B}}=y_{\bar{\partial}_{B}}$. Out of the modes marked with " $\star^{a}$ ", one vanishes when $\sigma$ is holomorphic. $y_{\bar{\partial}_{B}}=y+1, E_{\bar{\partial}_{B}}+\frac{1}{2}=E_{0}$. We included one of the fermionic modes, since it is the primary.

Short graviton, vector, and gravitino multiplets. When $f$ is holomorphic, the inequality $E_{\bar{\partial}_{B}} \geq y_{\bar{\partial}_{B}}$ is saturated. At the same time, several wave-functions vanish. Those remaining are marked with a " $\star$ " in table 8. Comparing the table with tables 12, 13, and 14 , we see that the vector multiplet $W$ remains unchanged, while the graviton multiplet shortens, becoming the short graviton multiplet. Out of the modes forming the long vector multiplet $A$, only

$$
S\left[f^{[0 ; q]}\right] \text { and } \pi\left[f^{[3 ; q-4 ;-]}\right]
$$

remain. Again, $E_{\bar{\partial}_{B}}=y_{\bar{\partial}_{B}}$ and one sees that these modes form a hypermultiplet as outlined in table 18. 


\begin{tabular}{|c|c|c|c|c|c|c|}
\hline Spin & Energy & Charge & Mass $^{2}$ & Name & Wave-f. & \\
\hline 1 & $E_{0}+\frac{3}{2}$ & $y-1$ & $4\left(E_{0}+3\right)\left(E_{0}+2\right)$ & $W$ & $\sigma^{[1 ; q-4]}$ & \\
1 & $E_{0}+\frac{3}{2}$ & $y+1$ & $4\left(E_{0}+3\right)\left(E_{0}+2\right)$ & $W$ & $\sigma^{[1 ; q]}$ & \\
1 & $E_{0}+\frac{1}{2}$ & $y-1$ & $4\left(E_{0}+2\right)\left(E_{0}+1\right)$ & $Z$ & $\sigma^{[2 ; q-4 ;+]}$ & \\
1 & $E_{0}+\frac{1}{2}$ & $y+1$ & $4\left(E_{0}+2\right)\left(E_{0}+1\right)$ & $Z$ & $\sigma^{[2 ; q ;+]}$ & \\
$\frac{1}{2}$ & $E_{0}$ & $y$ & $-E_{0}+\frac{3}{2}$ & $\lambda_{T}$ & $\sigma_{*}^{[1 / 2]}$ & $p$ \\
0 & $E_{0}+\frac{3}{2}$ & $y-1$ & $4\left(E_{0}+3\right)\left(E_{0}+2\right)$ & $\pi$ & $\sigma^{[3 ; q-4 ;+]}$ & \\
0 & $E_{0}+\frac{3}{2}$ & $y+1$ & $4\left(E_{0}+3\right)\left(E_{0}+2\right)$ & $\pi$ & $\sigma^{[3 ; q ;+]}$ & \\
0 & $E_{0}+\frac{1}{2}$ & $y-1$ & $4\left(E_{0}+2\right)\left(E_{0}+1\right)$ & $\phi$ & $\left.\sigma_{*}^{[2 ;} ; q-4\right]$ & \\
0 & $E_{0}+\frac{1}{2}$ & $y+1$ & $4\left(E_{0}+2\right)\left(E_{0}+1\right)$ & $\phi$ & $\sigma_{*}^{\left[2_{s} ; q\right]}$ & \\
\hline
\end{tabular}

Table 17. The gravitino multiplet $\chi^{-} \cdot y_{\bar{\partial}_{B}}=y+1, E_{\bar{\partial}_{B}}+\frac{5}{2}=E_{0}$. Again we include the fermionic primary.

The vector multiplet $Z$ shortens when $\chi$ is holomorphic. As shown in appendix C, all elements of $H_{\bar{\partial}_{B}}^{1,1}$ are primitive.

As mentioned in section 2.4, the holomorphic volume form $\Omega$ provides a map between scalars and $(2,0)$ forms, which allows us to understand the shortening of the vector multiplet $A$. Moreover, $\Omega$ allows us to map any $(0,1)$ form $\alpha$ to a $(2,0)$ form $\alpha\lrcorner \Omega$. The two-form is holomorphic if $\alpha$ is. However, $H_{\bar{\partial}_{B}}^{0,1}=0$ and thus any holomorphic $(2,0)$ form can be written as $\left.\bar{\partial}_{B} f\right\lrcorner \Omega$. From our considerations in section B.3 we know that holomorphic $(1,1)$ forms saturate the inequality

$$
\Delta_{2} \geq q_{2}\left(q_{2}+2\right)
$$

where we denote the charge of a two-from as $q_{2}$, to avoid confusion with that of $f$, which we still refer to as $q$. Furthermore, we know that the form $\left.\bar{\partial}_{B} f\right\lrcorner \Omega$ satisfies

$$
\left.\left.\left.\left.\left.\Delta_{2}\left(\bar{\partial}_{B} f\right\lrcorner \Omega\right)=(\delta+8) \bar{\partial}_{B} f\right\lrcorner \Omega, \quad £_{\xi}\left(\bar{\partial}_{B} f\right\lrcorner \Omega\right)=\imath(q+4) \bar{\partial}_{B} f\right\lrcorner \Omega=\imath q_{2} \bar{\partial}_{B} f\right\lrcorner \Omega .
$$

Assuming that the bound holds for generic two-forms, we find

$$
\delta+8 \geq(q+4)(q+6) .
$$

Solving for $\delta$ gives the shortening conditions for the vector multiplet $A$ :

$$
\left.\bar{\partial}_{B}\left(\bar{\partial}_{B} f\right\lrcorner \Omega\right)=0 \quad \text { and } \quad \delta=(q+2)(q+8)=4\left(y_{\bar{\partial}_{B}}+1\right)\left[\left(y_{\bar{\partial}_{B}}+1\right)+3\right] .
$$

Hence, even though none of the modes in the long vector multiplet $A$ is directly based on a two-form, the short vector multiplet $A$ corresponds to the cohomology group $H_{\bar{\partial}_{B}}^{2,0}$. As an aside, note that the relation between the $\Delta_{0}$ and $£_{\xi}$ eigenvalues in (4.10) is that of a holomorphic function with charge $q+2$.

The $\chi^{+}$and $\chi^{-}$gravitino multiplets are shown in tables 16 and 17 . When the $(1,0)$ form $\sigma$ is holomorphic, various modes disappear as discussed in B.2 and one obtains the 
short gravitino multiplet as well as a long gravitino multiplet $\chi^{-}$. We were not able to demonstrate the vanishing of the three-form $\sigma^{[3 ; q-4 ;-]}$ by direct manipulation of the differential forms. According to the superconformal algebra this mode should vanish. An analysis of the complete multiplet including fermions and Lichnerowicz modes should improve our understanding of the situation. One can also construct the gravitino multiplets in terms of $(0,1)$-forms $\tau$. As discussed in section B.2, these cannot be holomorphic, yet anti-holomorphic. When this happens, the $\chi^{-}$multiplet shortens while the length of the $\chi^{+}$multiplet remains unaffected. Once again the mode $\tau^{[3 ; q+4 ;+]}$ remains.

The massless graviton multiplet. When $f$ is constant, it is necessarily also holomorphic and antiholomorphic, so we can base the discussion on the holomorphic case. Additional wave-functions vanish and the short graviton multiplet shortens further to become the massless graviton multiplet.

Betti multiplets. Betti multiplets arise from a non-trivial second de Rham cohomology group $H_{d R}^{2}(Y)$. Given $\alpha \in H_{d R}^{2}(Y)$, we know that

$$
\bar{\partial}_{B} \alpha=\partial_{B} \alpha=£_{\xi} \alpha=\Delta \alpha=0 .
$$

Assuming $\alpha \in \Omega^{2,0}$, the arguments of the previous paragraphs imply that there is a scalar function $f$ such that $\left.\alpha=\bar{\partial}_{B} f\right\lrcorner \Omega$ and that $\Delta \alpha=(\delta+8) \alpha$ with $\Delta f=\delta f$. However, $\delta \geq 0$ which is in contradiction with $\alpha$ being harmonic. Hence, there are no harmonic $(2,0)$ forms. An analogue argument excludes $(0,2)$ forms. Similarly, we can exclude forms in $\Omega^{1} \wedge \eta$ since $d(\sigma \wedge \eta)=d \sigma \wedge \eta-2 \sigma \wedge J$ and $\sigma \wedge J=0$ has no solution. Turning to $(1,1)$ forms, we can ignore non-primitive forms since

$$
0=d(f J)=d f \wedge J
$$

implies that $f$ has to be a constant. In the end, the only candidates for Betti multiplets are the $(1,1)$ forms $\chi$ that are included in the vector multiplet $Z$. In other words,

$$
H_{d R}^{2}(Y) \subseteq H_{\bar{\partial}_{B}}^{1,1}
$$

For details on $H_{\bar{\partial}_{B}}^{1,1}$ see appendix C.

Finally, we can hazard a comment on Lichnerowicz modes in this case. The construction of table 15 assumes that one way to obtain such modes is via symmetric contraction of $(2,1)$ forms with $\bar{\Omega}$. When $\partial_{B} \chi=\bar{\partial}_{B} \chi=0$ the Lichnerowicz modes $\partial_{B} \chi \cdot \bar{\Omega}$ and $\bar{\partial}_{B} \chi \cdot \Omega$ vanish. Thus the multiplet has further shortening.

Hypermultiplets. As we discussed in the previous paragraphs, most modes of the long vector multiplet $A$ vanish when $f$ is holomorphic. The remaining modes form a hypermultiplet as shown in table 18.

Since the hypermultiplet consists of two scalars, a spinor and their complex conjugates, the primitive $(2,1)$ and $(1,2)$ forms of section B.4 also form hypermultiplets. Here, the assumption that these forms can be mapped to eigenmodes of the Lichnerowicz operator via

$$
\zeta^{[3 ; q]} \mapsto \zeta^{\left[2_{s} ; q-4\right]}=\zeta_{\kappa \lambda(\mu} \bar{\Omega}_{\nu)}{ }^{\kappa \lambda} \quad \text { and } \quad \vartheta^{[3 ; q]} \mapsto \vartheta^{\left[2_{s} ; q+4\right]}=\vartheta_{\kappa \lambda(\mu} \Omega_{\nu)}{ }^{\kappa \lambda}
$$




\begin{tabular}{|c|c|c|c|c|c|c|}
\hline Spin & Energy & Charge & Mass $^{2}$ & Name & Wave-f. & \\
\hline 0 & $y+1$ & $y-2$ & $4 y(y-1)$ & $\pi$ & $f^{[3 ; q-4 ;-]}$ & \\
0 & $y$ & $y$ & $4(y-2)(y-1)$ & $S$ & $f^{[0 ; q]}$ & $p$ \\
\hline 0 & $y+1$ & $-y+2$ & $4 y(y-1)$ & $\pi$ & $\bar{f}^{[3 ; q+4 ;+]}$ & \\
0 & $y$ & $-y$ & $4(y-2)(y-1)$ & $S$ & $\bar{f}^{[0 ; q]}$ & \\
\hline
\end{tabular}

Table 18. The hypermultiplet. $f^{[0 ; q]}$ and $f^{[3 ; q-4 ;-]}$ are the "survivors" from the long vector multiplet $A$ when $f$ is holomorphic. The other two modes are their complex conjugates. As to the normalization, $E_{0}=E_{\bar{\partial}_{B}}=y_{\bar{\partial}_{B}}=y$.

\begin{tabular}{|c|c|c|c|c|c|c|c|c|}
\hline Spin & Energy & Charge & Mass $^{2}$ & Name & Wave-f. & Name & Wave-f. & \\
\hline 0 & $y+1$ & $y-2$ & $4 y(y-1)$ & $\phi$ & $\zeta_{*}^{\left[2_{s} ; q-4\right]}$ & $\pi$ & $\vartheta^{[3 ; q]}$ & \\
0 & $y$ & $y$ & $4(y-2)(y-1)$ & $\pi$ & $\zeta^{[3 ; q]}$ & $\phi$ & $\vartheta_{*}^{\left[2_{s} ; q+4\right]}$ & $p$ \\
\hline 0 & $y+1$ & $-y+2$ & $4 y(y-1)$ & $\phi$ & $\bar{\zeta}_{*}^{\left[2_{s} ; q-4\right]}$ & $\pi$ & $\bar{\vartheta}_{*}^{[3 ; q]}$ & \\
0 & $y$ & $-y$ & $4(y-2)(y-1)$ & $\pi$ & $\bar{\zeta}^{[3 ; q]}$ & $\phi$ & $\bar{\vartheta}^{\left[2_{s} ; q+4\right]}$ & \\
\hline
\end{tabular}

Table 19. Hypermultiplets constructed from $(2,1)$ forms $\zeta$ and $(1,2)$ forms $\vartheta$.

is implied. The difference between the hypermultiplets in table 19 is the role reversal between the Lichnerowicz and three-form modes. As a result the charge of the $(1,2)$ multiplet is shifted when expressed in terms of the cohomology group $H_{\bar{\partial}_{B}}^{1,2}$.

\subsection{Previous work on Kaluza-Klein compactification}

The spectrum of Kaluza-Klein compactifications of eleven dimensional supergravity to anti-de Sitter spaces is best understood for coset spaces where harmonic analysis can be used. The multiplet tables in this paper are based on those in [16], thus our results agree with theirs.

Going beyond harmonic analysis, [33] and [37] consider consistent truncations of the eleven-dimensional theory. It is instructive to compare our findings with those of [33]. Considering the reduction of eleven-dimensional supergravity on seven-dimensional SU(3)structure manifolds, the authors of [33] assume the existence of the following real differentiable forms on $M_{7}$ : a one-form $\theta, n_{V}$ two-forms $\omega_{i}, 2 n_{H}$ three-forms $\alpha_{A}, \beta^{A}, n_{V}$ four-forms $\tilde{\omega}^{i}$, and a six-form $\tilde{\omega}^{0}$. They find that $n_{V}$ and $n_{H}$ correspond to the number of vector and hyper multiplets in the four-dimensional theory. The $\mathrm{SU}(3)$ structure is expressed in terms of the above forms as

$$
\eta=e^{V} \theta, \quad J=e^{-V} v^{i} \omega_{i}, \quad \Omega=e^{-\frac{3}{2} V}\left(Z^{A} \alpha_{A}-\mathcal{G}_{A} \beta^{A}\right) .
$$

Crucially, they impose a number of algebraic conditions on these forms. First of all, the forms are all annihilated by the vector $k$ defined by $\imath_{k} \theta=1$ from which it follows that - 
in the Sasaki-Einstein case - they are all elements of $\Omega^{p, q}$. Furthermore, they require

$$
\omega_{i} \wedge \tilde{\omega}^{j}=-\delta_{i}^{j} \tilde{\omega}^{0}, \quad \alpha_{A} \wedge \beta^{B}=-\delta_{A}^{B} \tilde{\omega}^{0} .
$$

Hence, one can think of $\tilde{\omega}^{j} \wedge \eta$ as the Hodge dual of $\omega_{i}$. The same goes for $\beta^{A} \wedge \eta$ and $\alpha_{A}$. Finally, the conditions

$$
\omega_{i} \wedge \alpha_{A}=\omega_{i} \wedge \beta^{A}=\tilde{\omega}^{i} \wedge \alpha_{A}=\tilde{\omega}^{i} \wedge \beta^{A}=\alpha_{A} \wedge \alpha_{B}=\beta^{A} \wedge \beta^{B}=0,
$$

are equivalent to our orthogonality conditions discussed in appendix B, since

$$
0=\alpha_{A} \wedge \tilde{\omega}^{i} \wedge \eta \sim \alpha_{A} \wedge \star \omega_{i}
$$

it follows that all three-forms $\alpha_{A}$ and $\beta^{A}$ are primitive. The same holds for $n_{V}-1$ two forms $\omega_{i}$, with the only exception given by the linear combination that defines $J$. In the Sasaki-Einstein case, the $2 n_{H}$ three-forms can be split into $n_{H}-1(2,1)$ forms, one $(3,0)$ form and their complex conjugates. Note that the forms $\omega_{i}$ cannot be $(2,0)$ or $(0,2)$ since they vanish under the action of $\Omega \wedge$ and $\bar{\Omega} \wedge$ and thus have to be of degree $(1,1)$.

Now, in our discussion we found one vector multiplet $Z$ for every primitive $(1,1)$ form, which corresponds to $n_{V}-1$ of the vector multiplets. We also find a vector multiplet $W$ for a constant scalar $f$, which corresponds to the non-primitive $(1,1)$ form $J$. This gives a total of $n_{V}$ vector multiplets. Of course, we find additional vector multiplets that do not appear in [33] since we also consider scalar fluctuations.

In section 4.2, we found two sources of hyper multiplets. First there are holomorphic scalars, which are equivalent to holomorphic $(3,0)$ forms. Second there are holomorphic primitive $(2,1)$ forms. We find more modes since our discussion includes scalar fluctuations.

The field content of [37] is that of a massless graviton multiplet together with a long vector multiplet. In the context of our paper, this corresponds to modes associated to constant scalars $f$.

\section{Conclusions}

The main result of this paper is the computation of the gravity superconformal index equation (2.3). The supergravity calculation of the index is based on Kaluza-Klein analysis of Sasaki-Einstein manifolds in section 4.

A slight question remains regarding our analysis of the short gravitino multiplet, where we were not able to show that the three-form $\sigma^{[3 ; q-4 ;-]}$ vanishes when $\sigma$ is holomorphic and the multiplet shortens. This is a shortcoming of our brute-force approach to constructing wave-functions, since one has to argue their vanishing one-by-one. An analysis of the supergravity variations relating the modes [15] is a starting point towards a more satisfactory derivation.

There is a number of further problems in the supergravity sector that we leave for future work. First, it is important to prove equation (4.5). Furthermore, there could possibly be additional shortening conditions. Finally, it would be very interesting to determine the spectrum of short multiplets and the superconformal index of more general supergravity 
backgrounds than compactifications on Sasaki-Einstein manifolds. This would give insight into theories whose holographic duals are even less well understood.

The superconformal index and the central charge $a$ of four-dimensional superconformal field theories are invariants of their associated Calabi-Yau 3-algebra [13, 38]. It would be very interesting to find a similar mathematical structure governing three-dimensional quiver Chern-Simons theories.

The superconformal index has proven to be a powerful tool in checking proposed dualities. While we have shown the equality of the field theory and gravity indices in several examples, much work remains to be done to show the equality with the field theory index for arbitrary geometries. All proposed field theory duals to Sasaki-Einstein seven manifolds can be tested by computing the field theory index $[8,9,12]$ and comparing it with the gravity index in equation (2.3).

Currently, there is no general procedure for constructing the field theory dual to a general Sasaki-Einstein seven manifold. For progress in this direction see [39-42]. It would be interesting to match the results of this paper with a direct field theory computation of the index for the theories proposed in [39-42]. ${ }^{9}$ We hope that the superconformal index will help to explore new holographic dualities.

\section{Acknowledgments}

The authors would like to thank Jyotirmoy Bhattacharya, Alexey Bondal, Sergey Galkin, and Yuji Tachikawa for helpful discussions. This work is supported in part by World Premier International Research Center Initiative (WPI Initiative), MEXT, Japan through the Institute for the Physics and Mathematics of the Universe, the University of Tokyo.

\section{A Conventions and useful expressions}

We list our conventions for the calculations in sections 4.2 and appendix B.

The Hodge star satisfies

$$
\begin{aligned}
\star \alpha_{\mu_{1} \ldots \mu_{d-p}} & =\frac{\sqrt{g}}{p !} \epsilon_{\mu_{1} \ldots \mu_{d-p}}{ }^{\nu_{1} \ldots \nu_{p}} \alpha_{\nu_{1} \ldots \nu_{p}}, \\
\star \star & =(-1)^{p(d-p)},
\end{aligned}
$$

the generalization of the interior product $\lrcorner$

$$
\begin{aligned}
\left.\alpha_{p}\right\lrcorner \beta_{q} & =\frac{1}{p !} \alpha^{\mu_{1} \ldots \mu_{p}} \beta_{\mu_{1} \ldots \mu_{p} \nu_{q-p} \ldots \nu_{q}} d x^{\nu_{q-p}} \otimes \cdots \otimes d x^{\nu_{q}}, \\
\star(\alpha \wedge \beta) & =\alpha\lrcorner \star \beta, \\
\left.\gamma_{1}\right\lrcorner\left(\alpha_{p} \wedge \beta_{q}\right) & \left.\left.=\left(\gamma_{1}\right\lrcorner \alpha_{p}\right) \wedge \beta_{q}+(-1)^{p} \alpha \wedge\left(\gamma_{1}\right\lrcorner \beta_{q}\right),
\end{aligned}
$$

\footnotetext{
${ }^{9}$ See also [43].
} 


\begin{tabular}{|cccc|}
\hline Scalars & & & \\
$\pi$ & $C_{3}$ & $Y_{[a b c]}$ & $Q^{2}+6 m Q+8 m^{2}$ \\
$\phi$ & $h_{a b}$ & $Y_{(a b)}$ & $\Delta_{L}-4 m^{2}$ \\
$S$ & $h^{a}{ }_{a}, C_{3}$ & $Y$ & $\Delta_{0}+44 m^{2}-12 m \sqrt{\Delta_{0}+9 m^{2}}$ \\
$\Sigma$ & $h^{a}{ }_{a}, C_{3}$ & $Y$ & $\Delta_{0}+44 m^{2}+12 m \sqrt{\Delta_{0}+9 m^{2}}$ \\
\hline 1-forms & & & \\
$A$ & $h_{m a}$ & $C_{m n a}$ & $\Delta_{1}+12 m^{2}-6 \sqrt{\Delta_{1}+4 m^{2}}$ \\
$W$ & $h_{m a}$ & $C_{m n a}$ & $\Delta_{1}+12 m^{2}+6 \sqrt{\Delta_{1}+4 m^{2}}$ \\
$Z$ & $C_{3}$ & $Y_{[a b]}$ & $\Delta_{2}$ \\
\hline Spin-2 & & & $\Delta_{0}$ \\
$h$ & $h_{\mu \nu}^{T T}$ & $Y$ & \\
\hline
\end{tabular}

Table 20. The anti-de Sitter mass relations [20, 21]. The table lists the $A d S_{4}$ field, its 11dimensional origin, the resulting 7 -dimensional wave-function, and finally the mass operator. Here, $m$ refers to the mass scale of the Freund-Rubin compactification, see (A.4), and is not to be confused with the mass of the bulk fields. In our conventions, $m=1$.

\begin{tabular}{|l|l|}
\hline Scalars & $\Delta_{ \pm}=\frac{1}{2}\left(3 \pm \sqrt{9+4 R^{2}\left(m^{2}-8\right)}\right)$ \\
p-Forms & $\Delta_{ \pm}=\frac{1}{2}\left(3 \pm \sqrt{(3-2 p)^{2}+4 R^{2} m^{2}}\right)$ \\
\hline
\end{tabular}

Table 21. Scaling dimension and anti-de Sitter mass. Here, $m$ is the mass of the relevant bulk field. For the origin of the slightly awkward -8 term in the scalar expression, see footnote 5 in [24]. Similarly, $R=1 / 2$.

and the various kinetic operators are given by

$$
\begin{aligned}
\Delta_{0} \alpha & =-\nabla^{\kappa} \nabla_{\kappa} \alpha, \\
\Delta_{1} \alpha_{\mu} & =\left(-\nabla^{\kappa} \nabla_{\kappa}+6\right) \alpha_{\mu}, \\
\Delta_{2} \alpha_{\mu \nu} & =-\nabla^{\kappa} \nabla_{\kappa} \alpha_{\mu \nu}-2 R_{\kappa \mu \lambda \nu} \alpha^{\kappa \lambda}+12 \alpha_{\mu \nu}, \\
Q \alpha_{\mu_{1} \mu_{2} \mu_{3}} & =(\star d \alpha)_{\mu_{1} \mu_{2} \mu_{3}}=\frac{\sqrt{g}}{4 !} \epsilon_{\mu_{1} \mu_{2} \mu_{3}}^{\nu_{1} \ldots \nu_{4}} d \alpha_{\nu_{1} \ldots \nu_{4}} .
\end{aligned}
$$

The operator $Q$ as defined here and $\bigotimes_{y}^{[1,1,1]}=M_{(1)^{3}}$ of [15] are related as $Q=4 \bigotimes_{y}^{[1,1,1]}$ since $\bigotimes_{y}^{[1,1,1]} Y^{a b c}=\frac{1}{4 !} \epsilon^{a b c d}{ }_{e f g} D_{d} Y^{e f g}$.

Turning to the properties of the Sasaki-Einstein links, we start with the Einstein condition

$$
R_{\mu \nu}=6 m^{2} g_{\mu \nu}
$$


Here, we chose $m=1$. Moreover, the spaces inherit from the Calabi-Yau cone the forms $\eta, J, \Omega$. The Reeb vector $\xi$ is given by

$$
\xi^{\mu}=g^{\mu \nu} \eta_{\nu}
$$

The forms satisfy

$$
\nabla_{\mu} \eta_{\nu}=J_{\mu \nu}, \quad \nabla_{\kappa} J_{\mu \nu}=-\eta_{\mu} g_{\kappa \nu}+\eta_{\nu} g_{\kappa \mu}, \quad \nabla_{\kappa} \Omega_{\lambda \mu \nu}=4 \imath \eta_{[\kappa} \Omega_{\lambda \mu \nu]}
$$

as well as

$$
\eta\lrcorner \eta=1, \quad \eta\lrcorner J=\eta\lrcorner \Omega=0 .
$$

It follows that $\eta, J, \Omega$ carry the charges $0,0,4$ under the Lie derivative along the Reeb vector $£_{\xi}$. Using the above relations, one can derive a number of useful contractions involving the Riemann tensor, such as

$$
\begin{aligned}
R_{\kappa \lambda \mu \nu} \eta^{\nu} & =g_{\kappa \mu} \eta_{\lambda}-g_{\lambda \mu} \eta_{\kappa} \\
J_{\mu}^{o} R_{\kappa \lambda \nu o}-J_{\nu}^{o} R_{\kappa \lambda \mu o} & =g_{\lambda \nu} J_{\kappa \mu}+g_{\kappa \mu} J_{\lambda \nu}-g_{\lambda \mu} J_{\kappa \nu}-g_{\kappa \nu} J_{\lambda \mu}, \\
2 R_{\mu \kappa \nu \lambda} \Omega^{\mu \nu \tau} & =R_{\mu \nu \kappa \lambda} \Omega^{\mu \nu \tau} \\
R_{\mu \nu \kappa \lambda} \Omega^{\kappa \lambda \tau} & =2 \Omega_{\mu \nu}{ }^{\tau} .
\end{aligned}
$$

Finally,

$$
\star 1=\frac{1}{3 !} J^{3} \wedge \eta, \quad \star \eta=\frac{1}{3 !} J^{3}, \quad \star J=\frac{1}{2} \eta \wedge J^{2}, \quad \star \Omega=-\imath \Omega \wedge \eta .
$$

As discussed in [13], the cotangent space can be decomposed as

$$
T Y^{*}=T^{1,0} Y^{*} \oplus T^{0,1} Y^{*} \oplus \mathbb{C} \eta
$$

where $T^{1,0} Y^{*}$ is the eigenspace of $\Pi^{+}$:

$$
\Pi^{ \pm}=\frac{1}{2}(g \mp \imath J-\eta \otimes \eta) .
$$

Thus, generic $k$-forms can decomposed as $(k=p+q)$

$$
\Omega^{k}(Y)=\Omega^{p, q} \oplus\left(\Omega^{p, q-1} \oplus \Omega^{p-1, q}\right) \wedge \eta .
$$

Furthermore, the exterior derivative can be decomposed as

$$
d=\partial_{B}+\bar{\partial}_{B}+\eta \wedge £_{\xi}
$$

The tangential Cauchy-Riemann operators satisfy

$$
\partial_{B} \bar{\partial}_{B}+\bar{\partial}_{B} \partial_{B}=-2 J \wedge £_{\xi}
$$




\section{B Details of the supergravity analysis}

In what follows, we will generally start with a eigenform $\alpha$ of the Hodge-de Rham operator, and use it to construct further eigenforms of $\Delta$ or $Q$. The procedure is quite straightforward $;^{10}$ one chooses a basis $v_{i}^{[\alpha ; p ; q]}$ and diagonalizes the matrix

$$
\Delta v_{i}^{[\alpha ; p ; q]}=M_{i j}^{[\alpha ; p ; q]} v_{j}^{[\alpha ; p ; q]} .
$$

Here, $\alpha$ labels the mode we started with, $p$ the form degree and $q$ the $R$-charge of the new modes.

\section{B.1 Wave functions constructed from scalars}

Consider a scalar eigenmode of the Hodge-de Rham operator with definite R-charge,

$$
\Delta f=\delta f, \quad £_{\xi} f=\imath q f=2 \imath \hat{y}_{0} f .
$$

At the level of one-forms, we consider the basis

$$
v_{i}^{[f ; 1 ; q]}=\left\{\eta f ; \imath\left(\partial_{B}-\bar{\partial}_{B}\right) f ; d f\right\}
$$

and find

$$
M^{[f ; 1 ; q]}=\left(\begin{array}{ccc}
\delta+12 & 2 & 0 \\
2 \delta & \delta & 2 \imath q \\
0 & 0 & \delta
\end{array}\right) .
$$

Diagonalization yields a gauge mode $d f$ as well as the forms $f^{[1 ; q ;+]}$ and $f^{[1 ; q ;-]}$.

Note that it is not possible to construct one-forms from $f$ with charge $q \pm 4$ - essentially, one would have to construct $(2,0)$ or $(0,2)$ forms out of $f$ and contract them with $\Omega$ or its conjugate. No such two-forms exist that are linear in $f$.

Proceeding to two-forms, we consider

$$
v_{i}^{[f ; 2 ; q]}=\left\{d \bar{\partial}_{B} f, \eta \wedge d f, f J, \eta \wedge\left(\partial_{B}-\bar{\partial}_{B}\right) f\right\}
$$

and find

$$
M^{[f ; 2 ; q]}=\left(\begin{array}{cccc}
\delta-2 q \imath[q(q+6)-\delta] & -2 \imath[q(q+6)-\delta] & 0 \\
4 \imath & \delta+2 q+8 & -4 q & 0 \\
0 & -2 & \delta+12 & 0 \\
0 & 2 q & -4 q & \delta+8 .
\end{array}\right)
$$

Diagonalization gives two gauge modes $-d f^{[1 ; q ; \pm]}$ - as well as $f^{[2 ; q ; a]}$ and $f^{[2 ; q ; b]}$.

At the level of two-forms we find the first modes with shifted charge. There is only one basis element in each case, so one can read off $M^{[f ; 2 ; q \pm 4]}$ from

$$
\begin{array}{ll}
\left.\left.\Delta\left(\bar{\partial}_{B} f\right\lrcorner \Omega\right)=(\delta+8) \bar{\partial}_{B} f\right\lrcorner \Omega, & \left.\left.£_{\xi}\left(\bar{\partial}_{B} f\right\lrcorner \Omega\right)=\imath(q+4)\left(\bar{\partial}_{B} f\right\lrcorner \Omega\right), \\
\left.\left.\Delta\left(\partial_{B} f\right\lrcorner \bar{\Omega}\right)=(\delta+8) \partial_{B} f\right\lrcorner \bar{\Omega}, & \left.\left.£_{\xi}\left(\partial_{B} f\right\lrcorner \bar{\Omega}\right)=\imath(q-4)\left(\partial_{B} f\right\lrcorner \bar{\Omega}\right) .
\end{array}
$$

\footnotetext{
${ }^{10}$ Some of the calculations get fairly involved. We found the Mathematica package xAct extremely helpful. [44, 45]
} 
Turning to three-forms, we study $Q=\star d$ instead of $\Delta$ with basis

$$
\left.\left.v_{i}^{[f ; 3 ; q]}=\{d f \wedge J, f \eta \wedge J,(d f\lrcorner J) \wedge J, \eta \wedge d(d f\lrcorner J\right)\right\},
$$

and

$$
M^{[f, 3, q]}=\left(\begin{array}{cccc}
0 & 0 & 0 & 0 \\
0 & 4 & -1 & 0 \\
-\imath q & -\delta & 0 & -1 \\
-2 \imath q & -2 \delta & 0 & -2
\end{array}\right) .
$$

Again, there are two gauge modes and $f^{[3 ; q ; \pm]}$

Now, there are two different ways to construct a charged $(3,0)$ form: $\left.\partial_{B}\left(\bar{\partial}_{B} f\right\lrcorner \Omega\right)$ and $f \Omega$. It is reasonable to assume that they are linearly related as long as $f$ is not holomorphic. Contracting both with $\bar{\Omega}$, one finds that

$$
\left.\partial_{B}\left(\bar{\partial}_{B} f\right\lrcorner \Omega\right)=-\frac{\delta-q(q+6)}{2} f \Omega .
$$

We still include both modes in the basis,

$$
\left.\left.\left.v_{i}^{[f ; 3 ; q+4]}=\left\{f \Omega, \eta \wedge\left(\bar{\partial}_{B} f\right\lrcorner \Omega\right),\left(\partial_{B}-\bar{\partial}_{B}\right)\left(\bar{\partial}_{B} f\right\lrcorner \Omega\right), d\left(\bar{\partial}_{B} f\right\lrcorner \Omega\right)\right\},
$$

and find

$$
M^{[f ; 3 ; q+4]}=\left(\begin{array}{cccc}
q+4 & \imath & 0 & 0 \\
0 & 2 & \imath & 0 \\
0 & -\imath(\delta+8) & 0 & q+4 \\
0 & 0 & 0 & 0
\end{array}\right) .
$$

There is one gauge mode and one additional mode with eigenvalue $q+4$ that is a remnant of the fact that our basis is not a basis. One also finds two eigenmodes $f^{[3 ; q+4 ; \pm]}$.

An identical calculation gives

$$
\left.\left.\left.v_{i}^{[f ; 3 ; q-4]}=\left\{f \bar{\Omega}, \eta \wedge\left(\partial_{B} f\right\lrcorner \bar{\Omega}\right),\left(\partial_{B}-\bar{\partial}_{B}\right)\left(\partial_{B} f\right\lrcorner \bar{\Omega}\right), d\left(\partial_{B} f\right\lrcorner \bar{\Omega}\right)\right\},
$$

and

$$
M^{[f ; 3 ; q-4]}=\left(\begin{array}{cccc}
-(q-4) & -\imath & 0 & 0 \\
0 & 2 & \imath & 0 \\
0 & -\imath(\delta+8) & 0 & q-4 \\
0 & 0 & 0 & 0
\end{array}\right) .
$$

One can also derive an equivalent relation for (B.10) with the anti-holomorphicity bound (B.17) appearing on the right hand side.

Shortening conditions. The spectrum for $f$ is actually bounded from below. Since

$$
\int \operatorname{vol} \bar{f} \Delta f=\int \operatorname{vol}\left[2\left|\bar{\partial}_{B} f\right|^{2}+q(q+6) \bar{f} f\right]
$$

it follows that

$$
\delta \geq q(q+6)
$$


with equality if and only if $f$ is holomorphic. Similarly, one finds that antiholomorphic $f$ corresponds to the bound

$$
\delta \geq q(q-6)
$$

The latter is to be expected, since complex conjugation acts on the charge as $q \mapsto-q$. Due to the Lichnerowicz obstruction [24], holomorphic, non-constant $f$ satisfy $q \geq 1$.

We introduce $E_{\bar{\partial}_{B}}$ via

$$
\delta=4 E_{\bar{\partial}_{B}}\left(E_{\bar{\partial}_{B}}+3\right)
$$

so (B.16) amounts to $E_{\bar{\partial}_{B}} \geq \frac{q}{2}$.

If $f$ is holomorphic the bound (B.15) is satisfied and many of the basis elements $v_{i}^{[f ; p]}$ vanish. So do a number of wave functions:

$$
f^{[1 ; q ;-]}, \quad f^{[2 ; q ; b]}, \quad f^{[2 ; q+4]}, \quad f^{[3 ; q ;-]}, \quad f^{[3 ; q+4 ;-]} \text { or } f^{[3 ; q+4 ;+]} .
$$

A number of remarks are in order here. First of all, neither of $f^{[2 ; q ; a, b]}$ vanishes, yet they coincide. We simply label the remaining mode $a$. Furthermore, out of the four forms in $v^{[f ; 3 ; q+4]}$, all except $f \Omega$ vanish. The latter is now an eigenform with eigenvalue $q+4$. Since $\delta=q(q+6)$, this agrees with the eigenvalues of $f^{[3 ; q+4 ;+]}$ for $q \geq 0$. For $q<-6$ however, it agrees with $f^{[3 ; q+4 ;-]}$.

Anti-holomorphy of $f$ leads to the vanishing of

$$
f^{[1 ; q ;+]}, \quad f^{[2 ; q ; b]}, \quad f^{[2 ; q-4]}, \quad f^{[3 ; q ;+]}, \quad f^{[3 ; q-4 ;+]} \text { or } f^{[3 ; q-4 ;-]} .
$$

Again, $f^{[2 ; q ; a, b]}$ conincide while now all elements of $f^{[f ; 3 ; q-4]}$ except $f \bar{\Omega}$ vanish. Since this has eigenvalue $q-4$, it corresponds to the - mode for $q \geq 6$ and to the + mode for $q \leq 0$.

The spectrum simplifies further when $f$ is constant. Now, we have $\delta=0=q$ while all modes except

$$
f^{[0 ; 0]}, \quad f^{[1 ; 0 ;+]}, \quad f^{[3 ; 0 ;+]}, \quad f^{[3 ;+4 ;+]}, \quad f^{[3 ;-4 ;+]}
$$

vanish. We ignore $f J=f^{[2 ; 0 ; a]}$ which is also an eigenmode, yet pure gauge.

Independent shortening conditions are given by

$$
\left.\bar{\partial}_{B}\left(\bar{\partial}_{B} f\right\lrcorner \Omega\right)=0,
$$

as well as $\left.\partial_{B}\left(\partial_{B} f\right\lrcorner \bar{\Omega}\right)=0$. See the discussion following equation (4.10) for details.

\section{B.2 Wave functions derived from one-forms}

We next consider one forms that were not covered in the discussion in section B.1. They need to be orthogonal to $\eta f, \partial_{B} f, \bar{\partial}_{B} f$. Moreover, such modes cannot be mapped to scalars. In total one needs to impose ${ }^{11}$

$$
\left.\eta\lrcorner \sigma=d^{\dagger} \sigma=\partial_{B}^{\dagger} \sigma=\bar{\partial}_{B}^{\dagger} \sigma=J\right\lrcorner d \sigma=0 .
$$

\footnotetext{
${ }^{11}$ Note that the primitivity condition on the exterior derivative $\left.-J\right\lrcorner d \sigma=0-$ is equivalent to the vanishing of $\partial_{B}^{\dagger} \sigma$ or $\bar{\partial}_{B}^{\dagger} \sigma$.
} 
Finally, $\sigma$ must not be exact in terms of $d, \partial_{B}, \bar{\partial}_{B}$. In what follows, we'll assume that

$$
\sigma^{[1 ; q]}=\sigma \in \Omega^{1,0}, \quad \Delta_{1} \sigma=\delta \sigma, \quad £_{\xi} \sigma=\imath q \sigma=2 \imath \hat{y}_{0} \sigma .
$$

There is actually a second one form with identical Hodge-de Rham eigenvalue $\delta$ :

$$
\left.\sigma^{[1 ; q-4]}=\partial_{B} \sigma\right\lrcorner \bar{\Omega} .
$$

At the level of two-forms with charge $q$, we consider

$$
v_{i}^{[\sigma ; 2 ; q]}=\left\{\sigma \wedge \eta, \imath\left(\partial_{B}-\bar{\partial}_{B}\right) \sigma-\eta \wedge \sigma, d \sigma\right\}
$$

and find

$$
M^{[\sigma ; 2 ; q]}=\left(\begin{array}{ccc}
\delta+10 & -2 & 0 \\
-2(\delta-5) & (\delta-2) & 2 \imath q \\
0 & 0 & \delta
\end{array}\right) .
$$

The two non-gauge eigenmodes are listed in table 9 .

Two-forms with shifted charge $q-4$ are constructed from the $(0,1)$ form $\left.\partial_{B} \sigma\right\lrcorner \bar{\Omega}$ :

$$
\left.\left.\left.v_{i}^{[\sigma ; 2 ; q-4]}=\left\{\eta \wedge\left(\partial_{B} \sigma\right\lrcorner \bar{\Omega}\right),\left(\partial_{B}-\bar{\partial}_{B}\right)\left(\partial_{B} \sigma\right\lrcorner \bar{\Omega}\right), d\left(\partial_{B} \sigma\right\lrcorner \bar{\Omega}\right)\right\} .
$$

Then

$$
M^{[\sigma ; 2 ; q-4]}=\left(\begin{array}{ccc}
\delta+8 & 2 \imath & 0 \\
-2 \imath \delta & \delta & 2 q-8 \\
0 & 0 & \delta
\end{array}\right) .
$$

Once again, there are two modes with eigenvalues $\delta+4 \pm 2 \sqrt{\delta+4}$.

Three-forms of charge $q$ can be constructed from

$$
v_{i}^{[\sigma ; 3 ; q]}=\left\{J \wedge \sigma, \eta \wedge\left(\partial_{B}-\bar{\partial}_{B}\right) \sigma, d\left(\partial_{B}-\bar{\partial}_{B}-2 \imath \eta \wedge\right) \sigma\right\} .
$$

Diagonalizing

$$
M^{[\sigma ; 3 ; q]}=\left(\begin{array}{ccc}
q & 1 & 0 \\
\delta-q^{2}+4 & -q & -\imath \\
0 & 0 & 0
\end{array}\right),
$$

one finds the modes $\sigma^{[3 ; q ; \pm]}$ with eigenvalues $\pm \sqrt{\delta+4}$ in table 9 .

For three-forms with charge $q-4$, one uses the same construction replacing $\sigma$ with $\left.\partial_{B} \sigma\right\lrcorner \bar{\Omega}$. Then

$$
\left.\left.\left.v_{i}^{[\sigma ; 3 ; q-4]}=\left\{J \wedge\left(\partial_{B} \sigma\right\lrcorner \bar{\Omega}\right), \eta \wedge\left(\partial_{B}-\bar{\partial}_{B}\right)\left(\partial_{B} \sigma\right\lrcorner \bar{\Omega}\right), d\left(\partial_{B}-\bar{\partial}_{B}-2 \imath \eta \wedge\right)\left(\partial_{B} \sigma\right\lrcorner \bar{\Omega}\right)\right\}
$$

and

$$
M^{[\sigma ; 3 ; q-4]}=\left(\begin{array}{ccc}
-q+4 & -1 & 0 \\
-\delta+q(q-8)+12 & q-4 & \imath \\
0 & 0 & 0
\end{array}\right) .
$$

The eigenvalues are again $\pm \sqrt{\delta+4}$. 
Shortening conditions. In principle, the Hodge-de Rham operator $\Delta_{1}$ should satisfy a bound similar to (B.16). By contracting with $J$, one can verify the equation

$$
\partial_{B} \bar{\partial}_{B} \sigma=\frac{\imath}{4}[\delta-q(q+4)] J \wedge \sigma
$$

which suggests

$$
\delta \geq q(q+4)
$$

In light of (B.35), we define $E_{\bar{\partial}_{B}}$ via

$$
\delta=4 E_{\bar{\partial}_{B}}\left(E_{\bar{\partial}_{B}}+2\right) .
$$

The holomorphy bound is again given by $E_{\bar{\partial}_{B}} \geq \frac{q}{2}$. Using similar methods one can show that antiholomorhpic $(0,1)$ forms $\tau$ satisfy

$$
\bar{\partial}_{B} \partial_{B} \tau=-\frac{\imath}{4}[\delta-q(q-4)] J \wedge \tau
$$

suggesting the bound

$$
\delta \geq q(q-4)
$$

If $\sigma$ is holomorphic, the basis elements in $v^{[\sigma ; 2,3 ; q]}$ become linearly dependent and the modes $\sigma^{[2,3 ; q ;-]}$ vanish. Since $H_{\partial_{B}}^{1,0}=0$, none of the shortening conditions affects the $(0,1)$-form $\left.\partial_{B} \sigma\right\lrcorner \bar{\Omega}$. If $\sigma$ is holomorphic, the associated $(0,1)$ form $\left.\partial_{B} \sigma\right\lrcorner \bar{\Omega}$ is not antiholomorphic. ${ }^{12}$ Since the fact that $\sigma$ is holomorphic implies

$$
\left.\left.\left.\left.\Delta\left(\partial_{B} \sigma\right\lrcorner \bar{\Omega}\right)=q(q+4) \partial_{B} \sigma\right\lrcorner \bar{\Omega}, \quad £_{\xi} \partial_{B} \sigma\right\lrcorner \bar{\Omega}=\imath(q-4) \partial_{B} \sigma\right\lrcorner \bar{\Omega},
$$

yet (B.38) demands that

$$
\left.\left.\Delta\left(\partial_{B} \sigma\right\lrcorner \bar{\Omega}\right)=(q-4)(q-8) \partial_{B} \sigma\right\lrcorner \bar{\Omega},
$$

which is clearly impossible.

\section{B.3 Wave functions derived from two-forms}

Proceeding to higher from degree, we consider two-forms that were not captured in the previous sections. See [32] for a similar, recent construction. Again we have to impose orthogonality to previously constructed forms while also demanding that these forms are not exact. Finally, it should not be possible to map them to forms of lower degree. Hence we consider forms $\chi$ satisfying

$$
\chi\lrcorner \Omega=\chi\lrcorner \bar{\Omega}=\chi\lrcorner J=\eta\lrcorner \chi=d^{\dagger} \chi=\partial_{B}^{\dagger} \chi=\bar{\partial}_{B}^{\dagger} \chi=0 .
$$

Note that $\chi$ is primitive.

$$
\chi^{[2 ; q]}=\chi \in \Omega_{p}^{1,1}, \quad \Delta_{2} \chi=\delta \chi, \quad £_{\xi} \chi=\imath \chi .
$$

\footnotetext{
${ }^{12}$ It cannot be holomorphic since $H_{\bar{\partial}_{B}}^{0,1}=0$.
} 
In order to construct three-forms, we use the basis

$$
v_{i}^{[\chi ; 3 ; q]}=\left\{\eta \wedge \chi,\left(\partial_{B}-\bar{\partial}_{B}\right) \chi, d \chi\right\} .
$$

Then the matrix

$$
M^{[\chi ; 3 ; q]}=\left(\begin{array}{ccc}
-2 & -\imath & 0 \\
\imath \delta & 0 & -q \\
0 & 0 & 0
\end{array}\right)
$$

yields two eigenmodes, listed in table 9 .

Shortening conditions. Contracting four-forms with $J$, one finds

$$
J\lrcorner(J \wedge \chi)=\chi, \quad J\lrcorner\left(d \bar{\partial}_{B} \chi\right)=\imath \frac{\delta-q(q+2)}{2} \chi .
$$

Thus

$$
\bar{\partial}_{B} \chi=0 \quad \Rightarrow \quad \delta=q(q+2) .
$$

Again we define $E_{\bar{\partial}_{B}}$ accordingly,

$$
\delta=4 E_{\bar{\partial}_{B}}\left(E_{\bar{\partial}_{B}}+1\right) \quad \Rightarrow \quad E_{\bar{\partial}_{B}} \geq \frac{q}{2} .
$$

Moreover, when $\chi$ is holomorphic, the basis elements in $v_{i}^{[\chi ; 3 ; q]}$ become linearly dependent. It turns out that the mode $\chi^{[3 ; q ;+]}$ vanishes.

When $d \chi=0, \chi$ is both holo- and anti-holomorphic with vanishing charge $q$. Again $\chi^{[3 ; q ;+]}$ vanishes and $q=\delta=0$. As we argue in section 4.2 , such forms are relevant for Betti multiplets.

\section{B.4 Additional three-form modes}

Finally, we consider the possibility of three-forms that have eluded us. The same considerations as in sections B.2 and B.3 yield that such forms are primitive, lie in $\Omega^{2,1} \oplus \Omega^{1,2}$. They are closed ${ }^{13}$ under $\partial_{B}$ and $\bar{\partial}_{B}$, co-closed under $d, \partial_{B}, \bar{\partial}_{B}$ and satisfy ${ }^{14} \zeta_{\kappa \lambda[\mu} \bar{\Omega}_{\nu]}{ }^{\kappa \lambda}=0$. Now, primitive, co-closed $(2,1)$ and $(1,2)$ forms are holomorphic if and only if they are antiholomorphic. Since $\partial_{B} \bar{\partial}_{B}+\bar{\partial}_{B} \partial_{B}=-2 J \wedge £_{\xi}$, consistency requires that they either carry no charge or are annihilated by the action of the Lefschetz operator $J \wedge$. If they carry no charge, they are closed under the exterior $d$. Assuming that

$$
\zeta^{[3 ; q]}=\zeta \in \Omega^{2,1}, \quad \vartheta^{[3 ; q]}=\vartheta \in \Omega^{1,2}, \quad £_{\xi} \zeta=\imath q \zeta, £_{\xi} \vartheta=\imath q \vartheta
$$

one finds that

$$
Q \zeta=\star d \zeta=-q \zeta \quad \text { and } \quad Q \vartheta=q \vartheta .
$$

\footnotetext{
${ }^{13}$ Otherwise $\zeta \in \Omega^{2,1}$ for example could be mapped to $\Omega^{1,1} \wedge \eta$ via $\star \bar{\partial}_{B} \zeta$.

${ }^{14}$ Equation (B.48) holds without imposing this. However, it appears necessary to impose this rule in order to avoid overlap with the modes constructed in section B.2 since
}

$$
\left(\zeta_{\kappa \lambda \mu} \bar{\Omega}_{\nu}{ }^{\kappa \lambda}\right) \Omega_{\rho}^{\mu \nu} d x^{\rho} \in \Omega^{1,0} .
$$




\section{Comments on Lefschetz decomposition and Kohn-Rossi cohomology}

We remind the reader that on Kähler manifolds, Lefschetz decomposition is the unique decomposition of $k$-forms in terms of primitive forms $k-2 h$ forms $a_{(h)}$ :

$$
\alpha=\sum_{h=0} a_{(h)} \wedge J^{h}
$$

On Kähler manifolds, the decomposition is compatible with cohomology.

Studying the standard proofs for the decomposition of forms [46, 47], it becomes clear that the proof of decomposition also holds in the Sasaki-Einstein case. I.e. given a generic $k$-form $\alpha$, there are unique forms $a_{(h)}^{\perp}, a_{(h)}^{\|}(h=0,1,2, \ldots)$ of degree $k-2 h$ and $k-2 h-1$ respectively and orthogonal to the Reeb vector $\xi$ such that

$$
\alpha=a_{(h)}^{\perp} \wedge J^{h}+\eta \wedge a_{(h)}^{\|} \wedge J^{h} .
$$

This decomposition is not compatible with de Rham cohomology as follows from the application of the exterior $d$ :

$$
d \alpha=\left(d a_{(h)}^{\perp}+2 a_{(h-1)}^{\|}\right) \wedge J^{h}-\eta \wedge d a_{(h)}^{\|} \wedge J^{h} .
$$

$d \alpha=0$ requires that the $\alpha_{(h)}^{\|}$are closed, yet the same cannot be said for the $\alpha_{(h)}^{\perp}$.

Let us turn to Kohn-Rossi cohomology. Here, we only consider elements of $\Omega_{Y}^{p, q}$ and thus all forms are annihilated by the action of $\eta\lrcorner$. Hence we can drop the $\eta \wedge$ terms in the decomposition (and thus also the $\perp$ subscripts). Acting with $\bar{\partial}_{B}$,

$$
\bar{\partial}_{B} \alpha=\sum_{h=0}^{\lfloor k / 2\rfloor} \bar{\partial}_{B} a_{(h)} \wedge J^{h}
$$

and we find that $\alpha$ is $\bar{\partial}_{B}$-closed if and only if the $a_{(h)}$ are. In what follows we will assume that this is the case (i.e. that $\alpha$ is $\bar{\partial}_{B}$-closed). Noting that $\alpha$ is a $£_{\xi}$ eigenmode if and only if the $a_{(h)}$ are, we assume also that $£_{\xi} a_{(h)}=\imath q a_{(h)}$ with $q \neq 0$. Finally, we will assume that $a_{(0)}=0$. Then, using (A.14), we find that $\alpha$ is $\bar{\partial}_{B}$-exact.

$$
\bar{\partial}_{B}\left(\frac{\imath}{2} q^{-1} \sum_{h=1}^{\lfloor k / 2\rfloor} \partial_{B} a_{(h)} \wedge J^{h-1}\right)=\sum_{h=1}^{\lfloor k / 2\rfloor} a_{(h)} \wedge J^{h}=\alpha .
$$

Thus we find the following result: All Kohn-Rossi cohomology classes $[\alpha]$ are either primitive or carry zero charge under $£_{\xi}$. This is a somewhat typical result for Sasaki-Einstein geometry. The U(1)-charge is an obstruction for the Lefschetz decomposition to behave as on Kähler manifolds. It seems reasonable to expect that for forms with zero charge Lefschetz decomposition extends to cohomology. With this we conjecture that Kohn-Rossi cohomology groups allow for the following decomposition, which makes use of the fact that the $£_{\xi}$ operator commutes with $\bar{\partial}_{B}, J \wedge$ and their adjoints:

$$
H_{\bar{\partial}_{B}}^{p, q}=\oplus_{\hat{q} \neq 0}\left[H_{\bar{\partial}_{B}}^{p, q}\right]_{\text {primitive }}^{£_{\xi}=i \hat{q}} \oplus_{k}\left[H_{\bar{\partial}_{B}}^{p-k, q-k}\right]^{£_{\xi}=0} .
$$


In the case of $(1,1)$ forms, it follows immediately that all forms are primitive, since the restriction of $H_{\bar{\partial}_{B}}^{0,0}$ to elements with zero charge is trivial:

$$
H_{\bar{\partial}_{B}}^{1,1}=\left[H_{\bar{\partial}_{B}}^{1,1}\right]_{\text {primitive }} \oplus\left[H_{\bar{\partial}_{B}}^{0,0}\right]^{£_{\xi}=0}=\left[H_{\bar{\partial}_{B}}^{1,1}\right]_{\text {primitive }} .
$$

A similar result holds for $(2,1)$ forms. Here, the question is whether holmorphic $(1,0)$ modes $\sigma$ with charge 0 contribute to $H_{\bar{\partial}_{B}}^{2,1}$ via $\sigma \wedge J$. Since $\sigma$ is holomorphic, the bound on the Laplace operator is satisfied. Since the charge is zero, $\sigma$ is harmonic and thus closed. Hence, $\partial_{B} \sigma=0$. Since $H_{\partial_{B}}^{1,0}=0$, there is a scalar $f$ such that $\sigma=\partial_{B} f$. Thus, all elements of $H_{\bar{\partial}_{B}}^{2,1}$ are primitive.

Interestingly, we can use the above construction to locally construct $(1,0)$ forms $j_{f}$ that satisfy $\bar{\partial}_{B} j_{f}=J$. Pick any scalar function $f$ that is holomorphic with respect to $\bar{\partial}_{B}$ and carries charge $q$. Then define

$$
j_{f}=\frac{\imath}{2} \partial_{B} \log f^{q^{-1}}
$$

Again, application of (A.14) gives the desired result $\bar{\partial}_{B} j_{f}=J$ locally.

\section{Cohomology using Borel-Weil-Bott}

The twisted cohomology groups of homogenous spaces can be computed by an extension of the Borel-Weil-Bott theorem [48, 49]. We summarize the results for complex projective space and the quadric $Q \in \mathbb{C P}^{4}$. For $\mathbb{C P}^{n}$ with ample line bundle $\mathcal{L}=\mathcal{O}(1)$, the ordinary cohomology groups are

$$
H^{p}\left(\mathbb{C P}^{n}, \Omega^{q}\right)= \begin{cases}\mathbb{C} & \text { if } \mathrm{p}=0 \\ 0 & \text { otherwise }\end{cases}
$$

For $\ell>0$, the twisted cohomology groups are

$$
H^{p}\left(\mathbb{C P}^{3}, \Omega^{q}(\ell)\right)= \begin{cases}\chi_{[\ell, 0,0]}^{A_{3}} & \text { if }(\mathrm{p}, \mathrm{q})=(0,0) \\ \chi_{[\ell-2,1,0]}^{A_{3}} & \text { if }(\mathrm{p}, \mathrm{q})=(0,1) \\ \chi_{[\ell-4,0,1]}^{A_{3}} & \text { if }(\mathrm{p}, \mathrm{q})=(0,2) \\ \chi_{[\ell-4,0,0]}^{A_{3}} & \text { if }(\mathrm{p}, \mathrm{q})=(0,3) \\ 0 & \text { otherwise. }\end{cases}
$$

The quadric $Q \in \mathbb{C P}^{4}$ is equipped with the line bundle $\mathcal{L}=\mathcal{O}_{Q}(1)$, which is the pullback of $\mathcal{O}_{\mathbb{C P}^{4}}(1)$ from the ambient projective space. Its cohomology groups are

$$
H^{p}\left(Q, \Omega^{q}\right)= \begin{cases}\mathbb{C} & \text { if } \mathrm{p}=0 \\ 0 & \text { otherwise }\end{cases}
$$


For $\ell>0$, the twisted cohomology groups are

$$
H^{p}\left(Q, \Omega^{q}(\ell)\right)= \begin{cases}\chi_{[\ell, 0]}^{\mathrm{SO}(5)} & \text { if }(\mathrm{p}, \mathrm{q})=(0,0) \\ \chi_{[\ell-2,2]}^{\mathrm{SO}(5)} & \text { if }(\mathrm{p}, \mathrm{q})=(0,1) \\ \chi_{[\ell-3,2]}^{\mathrm{SO}(5)} & \text { if }(\mathrm{p}, \mathrm{q})=(0,2) \\ \chi_{[\ell-3,0]}^{\mathrm{SO}(5)} & \text { if }(\mathrm{p}, \mathrm{q})=(0,3) \\ \mathbb{C} & \text { if }(p, q)=(1,2) \text { and } \ell=1 . \\ 0 & \text { otherwise. }\end{cases}
$$

Open Access. This article is distributed under the terms of the Creative Commons Attribution License (CC-BY 4.0), which permits any use, distribution and reproduction in any medium, provided the original author(s) and source are credited.

\section{References}

[1] G. Festuccia and N. Seiberg, Rigid supersymmetric theories in curved superspace, JHEP 06 (2011) 114 [arXiv:1105.0689] [INSPIRE].

[2] J. Kinney, J.M. Maldacena, S. Minwalla and S. Raju, An index for 4 dimensional super conformal theories, Commun. Math. Phys. 275 (2007) 209 [hep-th/0510251] [INSPIRE].

[3] C. Romelsberger, Counting chiral primaries in $N=1, D=4$ superconformal field theories, Nucl. Phys. B 747 (2006) 329 [hep-th/0510060] [INSPIRE].

[4] J. Bhattacharya, S. Bhattacharyya, S. Minwalla and S. Raju, Indices for superconformal field theories in 3, 5 and 6 dimensions, JHEP 02 (2008) 064 [arXiv:0801.1435] [INSPIRE].

[5] O. Aharony, O. Bergman, D.L. Jafferis and J. Maldacena, $N=6$ superconformal Chern-Simons-matter theories, M2-branes and their gravity duals, JHEP 10 (2008) 091 [arXiv:0806.1218] [INSPIRE].

[6] J. Bhattacharya and S. Minwalla, Superconformal indices for $N=6$ Chern Simons theories, JHEP 01 (2009) 014 [arXiv:0806.3251] [INSPIRE].

[7] S. Kim, The complete superconformal index for $N=6$ Chern-Simons theory, Nucl. Phys. B 821 (2009) 241 [Erratum ibid. B 864 (2012) 884] [arXiv:0903.4172] [INSPIRE].

[8] Y. Imamura and S. Yokoyama, Index for three dimensional superconformal field theories with general R-charge assignments, JHEP 04 (2011) 007 [arXiv: 1101.0557] [INSPIRE].

[9] S. Cheon, D. Gang, S. Kim and J. Park, Refined test of AdS $S_{4} / C F T_{3}$ correspondence for $N=2,3$ theories, JHEP 05 (2011) 027 [arXiv: 1102.4273] [INSPIRE].

[10] P. Merlatti, $M$ theory on $A d S_{4} \times Q^{111}$ : the complete $\operatorname{Osp}(2 \mid 4) \times \mathrm{SU}(2) \times \mathrm{SU}(2) \times \mathrm{SU}(2)$ spectrum from harmonic analysis, Class. Quant. Grav. 18 (2001) 2797 [hep-th/0012159] [INSPIRE].

[11] D. Gang, C. Hwang, S. Kim and J. Park, Tests of $A d S_{4} / C F T_{3}$ correspondence for $\mathcal{N}=2$ chiral-like theory, JHEP 02 (2012) 079 [arXiv:1111.4529] [INSPIRE].

[12] Y. Imamura, D. Yokoyama and S. Yokoyama, Superconformal index for large- $N$ quiver Chern-Simons theories, JHEP 08 (2011) 011 [arXiv:1102.0621] [INSPIRE]. 
[13] R. Eager, J. Schmude and Y. Tachikawa, Superconformal indices, Sasaki-Einstein manifolds and cyclic homologies, Adv. Theor. Math. Phys. 18 (2014) 129 [arXiv:1207.0573] [InSPIRE].

[14] A. Ceresole, G. Dall'Agata, R. D'Auria and S. Ferrara, $M$ theory on the Stiefel manifold and $3 D$ conformal field theories, JHEP 03 (2000) 011 [hep-th/9912107] [INSPIRE].

[15] R. D'Auria and P. Fré, Universal Bose-Fermi mass relations in Kaluza-Klein supergravity and harmonic analysis on coset manifolds with Killing spinors, Annals Phys. 162 (1985) 372 [INSPIRE].

[16] D. Fabbri, P. Fré, L. Gualtieri and P. Termonia, $M$ theory on $A d S_{4} \times M^{111}$ : the complete $\mathrm{Osp}(2 \mid 4) \times \mathrm{SU}(3) \times \mathrm{SU}(2)$ spectrum from harmonic analysis, Nucl. Phys. B 560 (1999) 617 [hep-th/9903036] [INSPIRE].

[17] C.N. Pope, Kähler manifolds and quantum gravity, J. Phys. A 15 (1982) 2455 [inSPIRE].

[18] J.J. Kohn and H. Rossi, On the extension of holomorphic functions from the boundary of a complex manifold, Ann. Math. 81 (1965) 451.

[19] S.S.T. Yau, Kohn-Rossi cohomology and its application to the complex Plateau problem. I, Ann. Math. 113 (1981) 67.

[20] M.J. Duff, B.E.W. Nilsson and C.N. Pope, Kaluza-Klein supergravity, Phys. Rept. 130 (1986) 1 [INSPIRE].

[21] H. Larsson, Kaluza-Klein mass spectra, p-branes and AdS/CFT, M.Sc. thesis, Linköping University, Göteborg, Sweden (2000).

[22] S. Gukov, Comments on $N=2$ AdS orbifolds, Phys. Lett. B 439 (1998) 23 [hep-th/9806180] [INSPIRE].

[23] Y. Nakayama, Index for orbifold quiver gauge theories, Phys. Lett. B 636 (2006) 132 [hep-th/0512280] [INSPIRE].

[24] J.P. Gauntlett, D. Martelli, J. Sparks and S.-T. Yau, Obstructions to the existence of Sasaki-Einstein metrics, Commun. Math. Phys. 273 (2007) 803 [hep-th/0607080] [InSPIRE].

[25] R. D'Auria and P. Fré, On the spectrum of the $N=2 \mathrm{SU}(3) \times \mathrm{SU}(2) \times \mathrm{U}(1)$ gauge theory from D $=11$ supergravity, Class. Quant. Grav. 1 (1984) 447 [InSPIRE].

[26] D. Fabbri et al., 3D superconformal theories from Sasakian seven manifolds: new nontrivial evidences for $A d S_{4} / C F T_{3}$, Nucl. Phys. B 577 (2000) 547 [hep-th/9907219] [INSPIRE].

[27] N. Benishti, D. Rodriguez-Gomez and J. Sparks, Baryonic symmetries and M5 branes in the $A d S_{4} / C F T_{3}$ correspondence, JHEP 07 (2010) 024 [arXiv: 1004.2045] [INSPIRE].

[28] D.A. Cox, J.B. Little and H.K. Schenck, Toric varieties, Graduate Series in Mathematics volume 124, American Mathematical Society, U.S.A. (2011).

[29] V.I. Danilov, The geometry of toric varieties, Uspekhi Mat. Nauk 33 (1978) 85.

[30] D. Martelli, J. Sparks and S.-T. Yau, Sasaki-Einstein manifolds and volume minimisation, Commun. Math. Phys. 280 (2008) 611 [hep-th/0603021] [INSPIRE].

[31] I.R. Shafarevich, Algebraic geometry V, Encyclopaedia of Mathematical Sciences volume 47, Springer-Verlag, Berlin, Germany (1999), Fano varieties; a translation of "Algebraic geometry V" (in Russian), A.N. Parshin and I.R. Shafarevich eds., Ross. Akad. Nauk, Vseross. Inst. Nauchn. i Tekhn. Inform., Moscow, Russia (1999). 
[32] K. Pilch and I. Yoo, On perturbative instability of Pope-Warner solutions on Sasaki-Einstein manifolds, JHEP 09 (2013) 124 [arXiv: 1305.0295] [INSPIRE].

[33] D. Cassani, P. Koerber and O. Varela, All homogeneous $N=2$ M-theory truncations with supersymmetric AdS4 vacua, JHEP 11 (2012) 173 [arXiv:1208.1262] [INSPIRE].

[34] D. Martelli and J. Sparks, $A d S_{4} / C F T_{3}$ duals from M2-branes at hypersurface singularities and their deformations, JHEP 12 (2009) 017 [arXiv:0909.2036] [INSPIRE].

[35] A. Ceresole, P. Fré and H. Nicolai, Multiplet structure and spectra of $N=2$ supersymmetric compactifications, Class. Quant. Grav. 2 (1985) 133 [INSPIRE].

[36] M. Billó, D. Fabbri, P. Fré, P. Merlatti and A. Zaffaroni, Shadow multiplets in $A d S_{4} / C F T_{3}$ and the super-Higgs mechanism: hints of new shadow supergravities, Nucl. Phys. B 591 (2000) 139 [hep-th/0005220] [inSPIRE].

[37] J.P. Gauntlett, S. Kim, O. Varela and D. Waldram, Consistent supersymmetric Kaluza-Klein truncations with massive modes, JHEP 04 (2009) 102 [arXiv:0901.0676] [INSPIRE].

[38] R. Eager, Equivalence of a-maximization and volume minimization, JHEP 01 (2014) 089 [arXiv: 1011.1809] [INSPIRE].

[39] M. Aganagic, A stringy origin of M2 brane Chern-Simons theories, Nucl. Phys. B 835 (2010) 1 [arXiv:0905.3415] [INSPIRE].

[40] F. Benini, C. Closset and S. Cremonesi, Chiral flavors and M2-branes at toric CY4 singularities, JHEP 02 (2010) 036 [arXiv:0911.4127] [INSPIRE].

[41] F. Benini, C. Closset and S. Cremonesi, Quantum moduli space of Chern-Simons quivers, wrapped D6-branes and AdS4/CFT3, JHEP 09 (2011) 005 [arXiv:1105.2299] [INSPIRE].

[42] C. Closset and S. Cremonesi, Toric Fano varieties and Chern-Simons quivers, JHEP 05 (2012) 060 [arXiv: 1201.2431] [INSPIRE].

[43] M. Fujita and T.-S. Tai, Eschenburg space as gravity dual of flavored $N=4$ Chern-Simons-matter theory, JHEP 09 (2009) 062 [arXiv: 0906. 0253] [INSPIRE].

[44] J. Martín-García, R. Portugal and L. Manssur, The Invar tensor package, Comput. Phys. Commun. 177 (2007) 640.

[45] J.M. Martín-García, D. Yllanes and R. Portugal, The Invar tensor package: differential invariants of Riemann, Comput. Phys. Commun. 179 (2008) 586 [arXiv:0802.1274] [INSPIRE].

[46] C. Voisin, Hodge theory and complex algebraic geometry, volume 1, Cambridge University Press, Cambridge U.K. (2008).

[47] D. Huybrechts, Complex geometry: an introduction, Springer, Germany (2005).

[48] D.M. Snow, Cohomology of twisted holomorphic forms on Grassmann manifolds and quadric hypersurfaces, Math. Ann. 276 (1986) 159.

[49] C. Dionisi and D. Faenzi, A simple vanishing theorem for twisted holomorphic forms on hermitian symmetric varieties, (2004). 